\title{
THE TENSILE PROPERTIES OF ALLOY STEELS AT ELE- VATED TEMPERATURES AS DETERMINED BY THE "SHORT-TIME" METHOD
}

\author{
By William Kahlbaum, ${ }^{1}$ R. L. Dowdell, and W. A. Tucker
}

\section{ABSTRACT}

By means of "short-time" tension tests a series of alloy steels has been tested at different elevated temperatures. The proportional limit was determined in each case. The materials tested were a plain carbon steel and commercial alloys of chromium, vanadium, and iron with and without additions of tungsten, silicon, or aluminum; also chromium-tungsten, nickel-molybdenum and several austenitic steels with and without tungsten. Metallographic study revealed very little intercrystalline weakness and no marked or significant change in structure resulting from the high temperature tests. The addition of tungsten increases the "structural stability" of the austenitic alloys. Hardness was determined as a further indication of tempering which might have occurred during the heating. A comparison of "short-time" test and "flow test" for pearlitic and austenitic steel is included.

\section{CONTENTS}

I. Introduction

II. Previous investigations

III. Test methods

IV. Materials

1. Short-time tension test 202

1. General

2. Microstructure

VI. Discussion

\section{INTRODUCTION}

During the last few years numerous papers have been published on the so-called "short-time" tension test and on the "long-time" or "flow" test for determining the tensile properties of alloys at elevated temperatures. Perhaps the main reason for the extensive investigations along this line is the fact that many types of industrial installations, such as steam boilers, turbines, cracking stills, extrusion liners, etc., are being gradually forced to higher working temperatues and pressures.

At present, engineers, in general, favor the "flow" or "creep" test for evaluating materials which must withstand service conditions involving continued loading at high temperatures, since this test 
duplicates to some extent service conditions. The tension specimen is maintained under constant load at some predetermined temperatures for a long period, often several hundred hours. From the data thus obtained, the stress required to produce a definite extension, for example, 0.1 or 1.0 per cent in 1,000 hours at any given temperature can be calculated. On the other hand, a period of only three or four hours is required for carrying out the "short-time" tension test at high temperatures. The most essential part of this test is the determination of the stress, for any given temperature, at which the material ceases to behave elastically.

The information available on the correlation of results from shorttime and from flow tests is rather limited. No conclusions that shorttime tests can entirely replace flow tests are warranted on the basis of available information. It is believed, however, that a large amount of valuable preliminary information can be obtained with the shorttime method with a great saving of time in the study and development of alloys for continuous service at elevated temperatures. In fact, since the advent of austenitic alloys for service at elevated temperatures there is still more reason for extended flow tests, such tests to be made for even longer periods of time than heretofore. As many austenitic alloys are metastable yet sluggish in their response to thermal treatment at temperatures in the vicinity of $1,200^{\circ}$ to $1,300^{\circ} \mathrm{F}$. $\left(600^{\circ}\right.$ to $700^{\circ}$ C.) it would not seem advisable to determine their characteristics by the short-time method only.

The group of materials reported upon in this paper includes both commercial alloy steels and some special alloys which had been made primarily to show the effects on the tensile properties of various alloying elements, such as silicon, chromium, nickel, vanadium, tungsten, molybdenum, and aluminum.

From a previous investigation ${ }^{2}$ results were available on the characteristics, as determined by the flow test, of three of the alloys used in the present work. These have been included in the present report, and form the basis of a comparison of the properties of identical materials as determined by the two methods. Two of, the alloys were austenitic in structure, whereas the other was representative of the martensitic class of this group of alloy steels.

All of the work reported here was carried out in accordance with the research associate plan ${ }^{3}$ in cooperation with The Midvale Co., Philadelphia, Pa., except the tests with the plain carbon steel. This work was done by E. L. Jones, guest worker for The International Combustion Engineering Corporation, New York, N. Y.

\section{PREVIOUS INVESTIGATIONS}

A very extensive bibliography on the effect of temperature on the properties of metals has been published. ${ }^{4}$ No attempt will be made in this report to give a detailed résumé of the previous work along

\footnotetext{
2 H. J. French, William Kahlbaum, and A. A. Peterson, Flow Characteristics of Special Fe-Ni-Cr alloys and some Steels at Elevated Temperatures, B. S. Jour. Research, 5, (RP192) p. 125; 1930.

${ }^{3}$ Bureau of Standards Circular No. 296.

4 Bibliography on Effect of Temperature on the Properties of Metals. (Published in 1928 by the A. S M. E., 29 West Thirty-ninth Street, New York N. Y. This is now being brought up to date.) Compiled by the Joint Research Committee of A. S. M. E. and A. S. T. M. on the Effect of Temperature on the Properties of Metals.
} 
this line. Some investigators $\begin{array}{lllllll}5 & 6 & 7 & 8 & 9 & 10 & \text { have given some results }\end{array}$ from short time tension tests and of flow tests of the same materials. In a previous bureau report ${ }^{11}$ the results of other investigators were given which showed that there were marked differences in the flow characteristics of similar steels reported by different laboratories. These differences depended on both the individual set-ups and on the interpretation of the data.

McVetty and Mochel ${ }^{12}$ have emphasized the extreme care which must be used with the short-time method if the results are to be of value as well as the necessary refinements in the design of furmace, control of temperature, measurement of extension, and measurement of stress. Within the last few years, an optical extensometer having an accuracy of measurement of approximately 0.000004 inch has become available. With such an instrument, it has been found that the apparent values of the proportional limit of metals are considerably less than those obtained with dial types, by means of which the extension can be read only to about 0.0002 inch. According to McVetty and Mochel:

A change in the accuracy of measurement of total elongation from 0.0002 inch to 0.000004 inch reduces the apparent value of proportional limit by about 17 per cent in the case of medium carbon steel and over 50 per cent in the case of the stainless iron.

The present tendency in both the short-time and flow tests of metals is toward higher sensitivity. The importance of extreme precautions of this kind has been further emphasized by McVetty. ${ }^{13}$

\section{TEST METHODS}

\section{SHORT-TIME TENSION TEST}

The method used for the short-time tension tests has been previously described and illustrated. ${ }^{14}$ All of the tests were made with a hydraulic machine, and the elongations were measured by means of a Martens' extensometer with its optical lever system as modified by Tuckerman ${ }^{15}$ and as described in Letter Circular No. 238, issued by the Bureau of Standards.

The thermocouple used for measuring the temperature of the test bar was mounted in the fillet of the standard 0.505-inch diameter bar. Before attempting to obtain any of the data for the stress-strain curve, the furnace was held at the desired temperature, by manual control,

B J. H. S. Dickenson, Some Experiments on the Flow of Steels at a Low Red Heat with a Note on the Scaling of Heated Steels, J. Iron \& Steel Inst., 106, p. 103; 1922.

${ }^{6}$ H. J. French and W. A. Tucker, Flow in a Low-Carbon Steel at Various Temperatures, B. S. Tech. Paper No. 296, p. 630; 1925 .

7 T. D. Lynch, N. L. Mochel, P. G. McVetty, The Tensile Properties of Metals at High Temperatures, Proc. Am. Soc. Tesing Materials, 25, pt. 2, p. $5 ; 1925$.

8 H. J. French, Methods of Test in Relation to Flow in Stecls at Various Tempcratures, Proc. Am. Soc.

Testing Materials, 26, pt. 2, p. 7; 1926; also Eng. News Record, 97, p. 22.

A. Pomp and A. Dahmen, Entwicklung einer abgekurtzen plufverfahrens zur entmittlung der dauerstandfostigkeit von stahl bei erhohten temperaturen, Mitteil. a. d. Kaiser-Wilhelm Inst. f. Eisenforschung, Dusseldorf, 9, No. 3, 1927; also Stahl u. Eisen, 47, p. 414; 1927.

${ }_{10}$ L. W. Spring, J. J. Kanter, "Long-Time" or "Flow" Tests of Carbon Steels at Various Temperatures with Particular Reference to Stresses below the Proportional Limit, Valve World, p. 260, August, 1928; also in a shorter form in Proc. Am. Soc. Testing Material 25, pt. 2, pp. 80-116; 1928.

11 H. J. French, H. C. Cross, and A. A. Peterson, Creep in Five Steels at Different Temperatures, B. S.

Tech. Paper No. 362; 1928.

12 P. G. McVetty and N. L. Mochel, The Tensile Properties of Stainless Iron and Other Alloys at Elevated Temperatures, Trans. Am. Soc. Steel Treat., 11, pp. 73-100; 1927.

${ }_{13}$ P. G. McVetty, Testing of Materials at Elevated Temperatures, Proc. Am. Soc. Testing Mate-

rials, 28, pt. 2, p. 60; 1928.

is See footnotes 8 and 11 .

26 Engineer physicist, Bureau of Standards. 
for a period of two hours. The temperature variation from the center to the extremities of the gage length of the specimen was approximately $13^{\circ} \mathrm{F}$. $\left(7^{\circ} \mathrm{C}\right.$.) It was possible to run only one shorttime test a day owing to the necessity of exercising extreme care in setting up and adjusting the extensometer. The actual duration of the average test was about one-half hour. The accuracy of the extensometer was 0.000004 inch per inch of extension while the load measurements were accurate to within one-half of 1 per cent.

\section{2. "LONG-TIME" TENSION TEST-"FLOW" TEST}

The equipment used in carrying out the flow tests has been previously described. ${ }^{16,17}$ The load was applied by weights at the ends of - levers. The diameter of the test bar was 0.250 inch and the gage length, 2 inches. A shallow groove was cut close to the fillet at each end of the specimen, in which was secured a ring of platinum wire (32 B. \& S. gage) used as a reference mark in measuring the extension of the bar. A cathetometer whose smallest direct reading was 0.00039 inch was used to measure the distance between reference marks at periodic intervals. The extension was calculated in inches per inch of gage length.

The automatic temperature controllers on the furnaces were actuated by means of thermocouples located close to the furnace windings, the temperature was maintained within a range of $\pm 10^{\circ} \mathrm{F}$. $\left( \pm 5.5^{\circ} \mathrm{C}.\right)$.

The duration of the flow tests was from 300 to 400 hours, and from the results were calculated the limits of stress producing a total extension or "flow" of both 0.1 per cent and 1 per cent in 1,000 hours.

\section{MATERIALS}

A wide variety of ferrous alloys was tested. The chemical composition and treatment of these alloys are given in Table 1 . The plain carbon steel ( 0.45 per cent carbon) listed is typical of material widely used for boiler drums in steam power plants. The remainder of the alloys were made, treated, and analyzed by The Midvale Co. with the exception of the two foreign alloys, FS-1 and FS-2. The list of alloys covers a rather wide range of composition, and they were made up primarily to show the effects of various alloying elements on the tensile properties at elevated temperatures as measured by the shorttime method. All of the alloys were in the wrought condition, either forged or rolled, after which operation many had been heat-treated as shown in Table 1. 


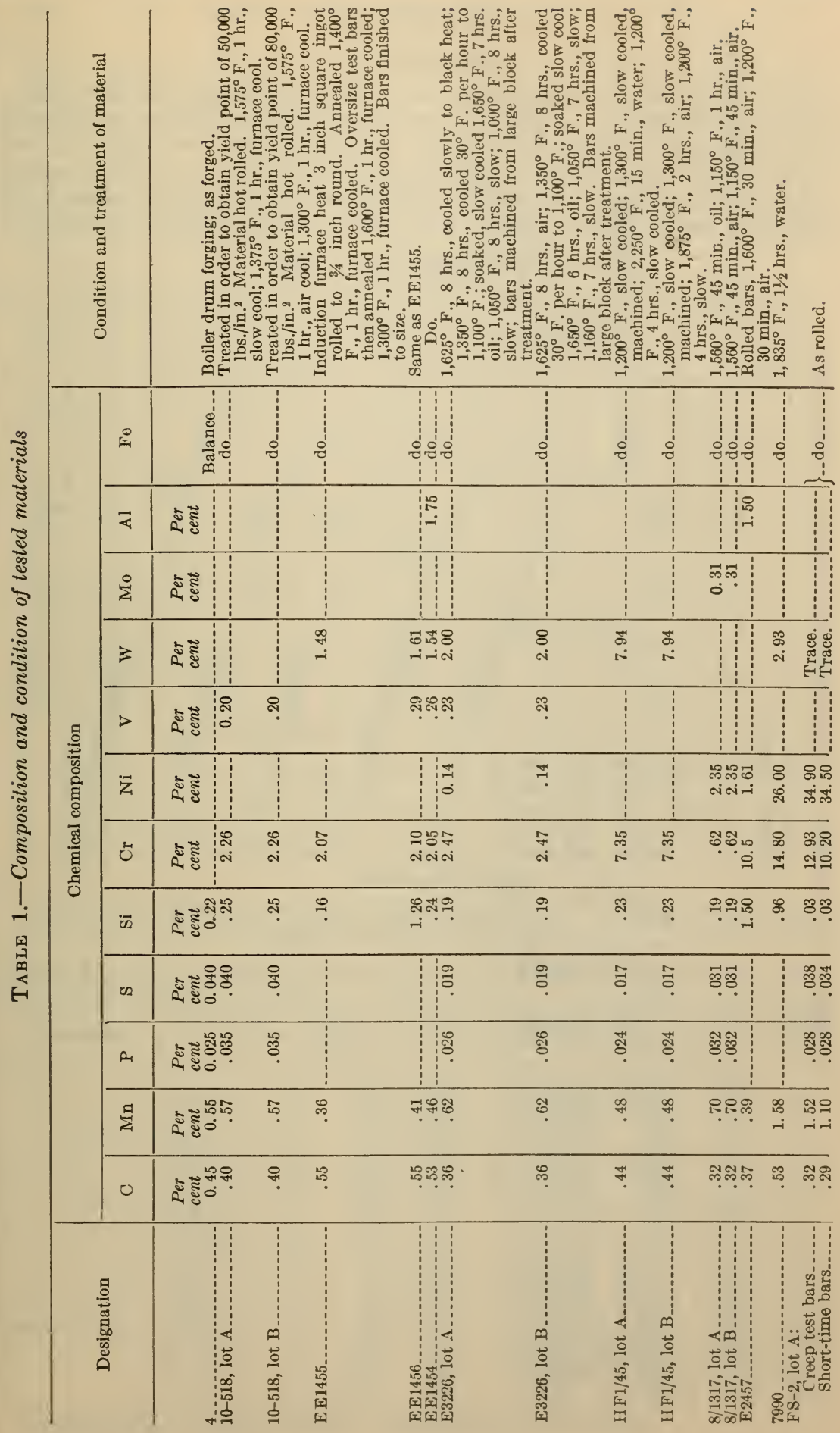




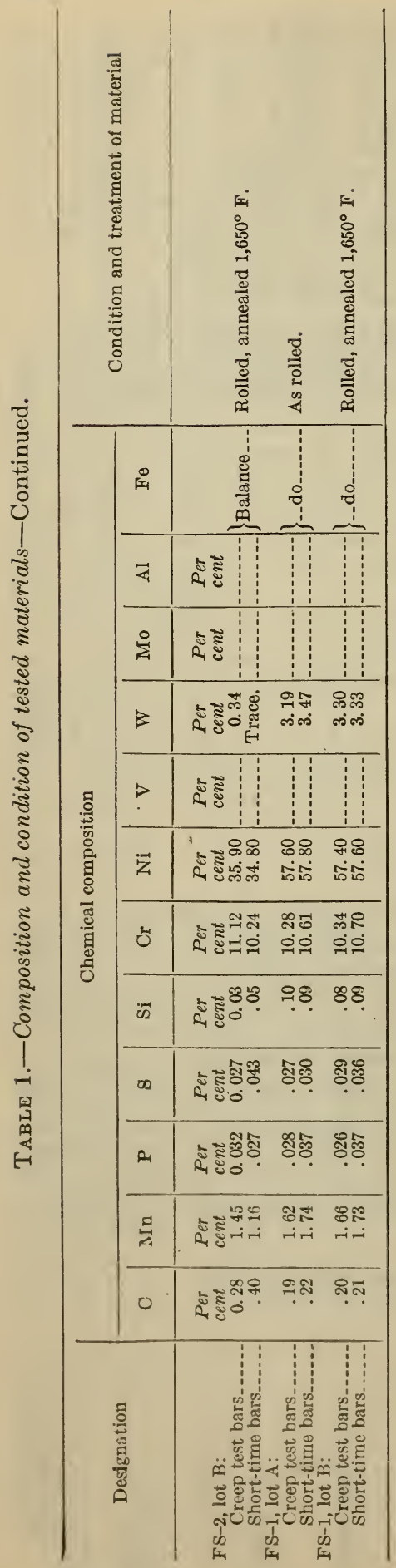




\section{RESULTS}

\section{GENERAL}

The tensile properties of all of the materials as determined by the short-time testing method are given in Table 2. Stress-strain curves for a number of representative alloys of different composition are given in Figures 1 to 11, inclusive. Figure 12 shows a comparison of the results of those alloys for which both proportional limits from the short-time tension tests and the flow characteristics were available.

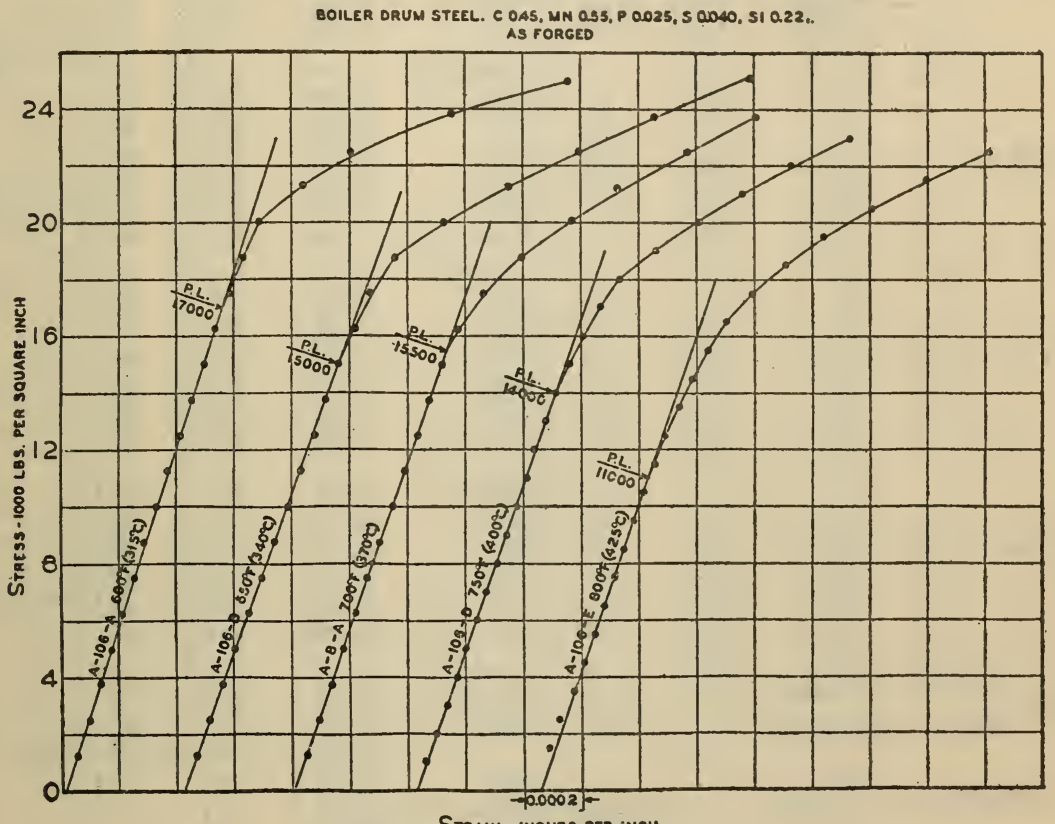

STRAIN - INCHES PER INCH

FIGURE 1.-Stress-strain curves of medium carbon steel (boilerdrum) tested in tension at different temperatures

TABLE 2.-Tensile properties of materials tested in Table 1 at different temperatures

\begin{tabular}{|c|c|c|c|c|c|c|c|}
\hline Designation & Specimen No. & $\begin{array}{c}\text { Test } \\
\text { tempera- } \\
\text { ture }\end{array}$ & $\begin{array}{l}\text { Proportional } \\
\text { limit }\end{array}$ & $\begin{array}{l}\text { Tensile } \\
\text { strength }\end{array}$ & $\begin{array}{l}\text { Elonga- } \\
\text { tion in } 2 \\
\text { inches }\end{array}$ & $\begin{array}{l}\text { Reduc- } \\
\text { tion of } \\
\text { area }\end{array}$ & $\begin{array}{c}\text { Brinell } \\
\text { hardness } \\
\text { No. }\end{array}$ \\
\hline $\int^{(1)}$. & $\left\{\begin{array}{l}A-4-A- \\
A-4-B- \\
A-4-C- \\
A-4-D= \\
A-4-E \\
A-106-A \\
A-106-B \\
A-8-A \\
A-106-D \\
A-106-E\end{array}\right.$ & $\begin{array}{r}\circ F \text {. } \\
600 \\
650 \\
700 \\
750 \\
800 \\
600 \\
650 \\
700 \\
750 \\
800\end{array}$ & $\begin{array}{l}\text { Lbs./in. }{ }^{2} \\
16,500 \\
15,500 \\
16,500 \\
15,000 \\
12,000 \\
17,000 \\
15,000 \\
15,500 \\
14,000 \\
11,000\end{array}$ & $\begin{array}{r}\text { Lbs./in. }{ }^{2} \\
78,700 \\
75,850 \\
74,000 \\
68,500 \\
62,250 \\
80,000 \\
77,500 \\
71,500 \\
66,000 \\
67,000\end{array}$ & $\begin{array}{r}\text { Per cent } \\
22.0 \\
21.5 \\
24.0 \\
23.5 \\
23.0 \\
27.0 \\
26.0 \\
22.0 \\
26.5 \\
24.0\end{array}$ & $\begin{array}{r}\text { Per cent } \\
24.8 \\
38.9 \\
44.5 \\
51.7 \\
50.8 \\
38.1 \\
42.7 \\
48.8 \\
53.0 \\
49.1\end{array}$ & $\begin{array}{l}0 \\
-1 \\
-1\end{array}$ \\
\hline $10-518$, lot $\mathrm{A}$ & $\left\{\begin{array}{l}\mathrm{T}-19 \\
\mathrm{~T}-15- \\
\mathrm{T}-3136 \\
\mathrm{~T}-3127- \\
\mathrm{T}-125 \\
\mathrm{~T}-3139 \\
\mathrm{~T}-3113 . \\
\mathrm{T}-11 .= \\
\mathrm{T}-12 \mathrm{1}\end{array}\right.$ & $\begin{array}{r}70 \\
70 \\
550 \\
550 \\
800 \\
800 \\
1,000 \\
1,000 \\
1,200\end{array}$ & $\begin{array}{r}32,500 \\
30,000 \\
24,000 \\
22,500 \\
18,500 \\
17,750 \\
6,000 \\
4,400 \\
500\end{array}$ & $\begin{array}{l}84,250 \\
82,250 \\
80,500 \\
75,000 \\
73,750 \\
73,750 \\
39,000 \\
40,000 \\
26,750\end{array}$ & $\begin{array}{l}33.0 \\
31.0 \\
22.5 \\
27.0 \\
26.5 \\
25.0 \\
36.5 \\
37.5 \\
41.0\end{array}$ & $\begin{array}{l}68.2 \\
70.8 \\
66.8 \\
69.8 \\
68.8 \\
67.7 \\
88.3 \\
85.1 \\
92.8\end{array}$ & 170 \\
\hline
\end{tabular}

1 Specimens of the first group of steel No. 4 were transverse test specimens.

Specimens of the second group of steel No. 4 were longitudinal test specimens. 
TABLE 2.-Tensile properties of materials tested in Table 1 at different temperatures-Continued

\begin{tabular}{|c|c|c|c|c|c|c|c|}
\hline Designation & Specimen No. & $\begin{array}{c}\text { Test } \\
\text { tempera- } \\
\text { ture }\end{array}$ & $\begin{array}{l}\text { Proportional } \\
\text { limit }\end{array}$ & $\begin{array}{l}\text { Tensile } \\
\text { strength }\end{array}$ & $\begin{array}{l}\text { Elonga- } \\
\text { tion in } 2 \\
\text { inches }\end{array}$ & $\begin{array}{l}\text { Reduc- } \\
\text { tion of } \\
\text { area }\end{array}$ & $\begin{array}{c}\text { Brinell } \\
\text { hardness } \\
\text { No. }\end{array}$ \\
\hline 10-518, lot B. & $\left\{\begin{array}{l}\mathrm{T}-261 \\
\mathrm{~T}-3109 \\
\mathrm{~T}-3104 \\
\mathrm{~T}-253 \\
\mathrm{~T}-276 \\
\mathrm{~T}-257 \\
\mathrm{~T}-290\end{array}\right.$ & $\begin{array}{r}\circ F . \\
70 \\
70 \\
550 \\
800 \\
1,000 \\
1,000 \\
1,200\end{array}$ & $\begin{array}{c}\text { Lbs./in. }{ }^{2} \\
63,500 \\
58,500 \\
40,000 \\
21,500 \\
8,750 \\
8,375 \\
600\end{array}$ & $\begin{array}{r}\text { Llbs./in. }{ }^{2} \\
100,650 \\
102,500 \\
91,500 \\
83,750 \\
61,500 \\
51,000 \\
37,250\end{array}$ & $\begin{array}{r}\text { Per cent } \\
27.0 \\
27.5 \\
21.0 \\
24.0 \\
27.5 \\
30.0 \\
32.0\end{array}$ & $\begin{array}{r}\text { Per cent } \\
66.1 \\
66.8 \\
68.2 \\
68.6 \\
80.4 \\
87.5 \\
91.6\end{array}$ & \begin{tabular}{r}
201 \\
\hdashline-1 \\
-1. \\
-1
\end{tabular} \\
\hline EE1455 & $\left\{\begin{array}{l}3-- \\
4- \\
5- \\
6- \\
3- \\
2- \\
7-- \\
8-\end{array}\right.$ & $\begin{array}{r}70 \\
850 \\
850 \\
950 \\
950 \\
1,050 \\
1.050 \\
1,200 \\
1,200\end{array}$ & $\begin{array}{c}66,000 \mathrm{E} . \mathrm{L} .{ }^{3} \\
25,000 \\
25,000 \\
16,500 \\
18,000 \\
9,800 \\
10,500 \\
2,000 \\
4,000\end{array}$ & $\begin{array}{r}111,500 \\
90,000 \\
88,500 \\
79,500 \\
80,000 \\
63,000 \\
62,500 \\
52,000 \\
47,500\end{array}$ & $\begin{array}{l}21.2 \\
23.5 \\
24.0 \\
25.0 \\
25.0 \\
31.5 \\
30.0 \\
25.0 \\
27.5\end{array}$ & $\begin{array}{l}52.2 \\
62.7 \\
62.2 \\
66.5 \\
66.5 \\
79.6 \\
79.2 \\
78.5 \\
83.2\end{array}$ & $\begin{array}{l}197 \\
- \\
- \\
-1 \\
-1\end{array}$ \\
\hline EE1456 & $\left\{\begin{array}{l}--- \\
2-- \\
1-- \\
3-- \\
6-- \\
4-- \\
5-- \\
7- \\
8-\end{array}\right.$ & $\begin{array}{r}70 \\
850 \\
850 \\
950 \\
950 \\
1,050 \\
1,050 \\
1,200 \\
1,200\end{array}$ & $\begin{array}{l}75,000 \text { E. L. } \\
28,000 \\
50,000 \\
24,000 \\
24,000 \\
18,000 \\
16,000 \\
8,000 \\
8,000\end{array}$ & $\begin{array}{r}122,000 \\
99,000 \\
92,300 \\
85,000 \\
81,500 \\
65,000 \\
63,000 \\
44,000 \\
53,000\end{array}$ & $\begin{array}{l}21.2 \\
25.0 \\
24.5 \\
28.0 \\
30,0 \\
32.0 \\
31.5 \\
33.5 \\
29.0\end{array}$ & $\begin{array}{l}53.1 \\
63.0 \\
59.3 \\
71.3 \\
73.7 \\
82.2 \\
80.1 \\
85.8 \\
86.3\end{array}$ & 219 \\
\hline EE1454.. & & $\begin{array}{r}70 \\
850 \\
850 \\
950 \\
950 \\
1,050 \\
1,200 \\
1,200\end{array}$ & $\begin{array}{l}66,000 \text { E. L. }{ }^{3} \\
29,000 \\
30,000 \\
24,000 \\
22,000 \\
13,000 \\
8,500 \\
9,000\end{array}$ & $\begin{array}{r}104,500 \\
89,000 \\
89,500 \\
82,200 \\
78,500 \\
68,000 \\
50,500 \\
48,000\end{array}$ & $\begin{array}{l}17.7 \\
21.5 \\
21.5 \\
22.5 \\
23.5 \\
28.0 \\
32.5 \\
33.0\end{array}$ & $\begin{array}{l}34.4 \\
54.0 \\
54.3 \\
59.6 \\
62.7 \\
74.1 \\
83.7 \\
84.9\end{array}$ & 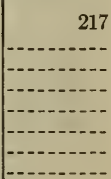 \\
\hline E3226 $\left\{\begin{array}{l}\text { Lot A. } \\
\text { Lot B. }\end{array}\right.$ & $\left\{\begin{array}{l}\mathrm{S}-350 \\
\mathrm{~S}-350\end{array}\right.$ & $\begin{array}{r}70 \\
1,000 \\
70 \\
1,000\end{array}$ & $\begin{array}{l}152,000 \text { E. L. }{ }^{3} \\
32,500 \\
96,000 \text { E. L. } \\
28,000\end{array}$ & $\begin{array}{r}201,000 \\
113,500 \\
132,000 \\
84,000\end{array}$ & $\begin{array}{l}15.0 \\
15.0 \\
17.0 \\
22.0\end{array}$ & $\begin{array}{l}52.8 \\
56.4 \\
52.0 \\
73.4\end{array}$ & \\
\hline HF $1 / 45$, lot A. & $\left\{\begin{array}{l}\mathrm{F}-1 / 4 \\
\mathrm{~F}-1 / 4\end{array}\right.$ & $\begin{array}{r}70 \\
1,000 \\
1,000\end{array}$ & $\begin{array}{l}75,800 \\
44,000 \\
46,300\end{array}$ & $\begin{array}{l}227,800 \\
163,400 \\
159,400\end{array}$ & $\begin{array}{l}8.0 \\
6.0 \\
6.5\end{array}$ & $\begin{array}{l}17.5 \\
11.4 \\
11.4\end{array}$ & \\
\hline $\mathrm{HF} 1 / 45$, lot $\mathrm{B}$ & $\left\{\begin{array}{l}\mathrm{F}-1 / 45-1 \\
\mathrm{~F}-1 / 45-2\end{array}\right.$ & $\begin{array}{r}70 \\
1,000 \\
1,150\end{array}$ & $\begin{array}{l}70,000 \text { E. L. }{ }^{3} \\
21,000 \\
13,500\end{array}$ & $\begin{array}{r}153,000 \\
95,000 \\
73,000\end{array}$ & $\begin{array}{l}12.0 \\
15.0 \\
21.5\end{array}$ & $\begin{array}{l}36.9 \\
49.1 \\
66.5\end{array}$ & \\
\hline $8 / 1317, \operatorname{lot} \mathrm{A}$. & $\left\{\begin{array}{l}1 \mathrm{~A}+- \\
1 \mathrm{E}- \\
1 \mathrm{C}- \\
1 \mathrm{D}= \\
1 \mathrm{~F}-\end{array}\right.$ & $\begin{array}{r}70 \\
800 \\
800 \\
900 \\
900 \\
1,000\end{array}$ & $\begin{array}{c}126,000 \text { E. L. } \\
52,500 \\
52,500 \\
35,000 \\
34,000 \\
12,000\end{array}$ & $\begin{array}{r}156,000 \\
123,000 \\
132,000 \\
109,500 \\
111,500 \\
98,000\end{array}$ & $\begin{array}{l}17.0 \\
16.5 \\
15.0 \\
17.5 \\
17.0 \\
20.0\end{array}$ & $\begin{array}{l}59.4 \\
61.1 \\
57.5 \\
69.7 \\
68.3 \\
75.6\end{array}$ & 325 \\
\hline $8 / 1317$, lot $\mathrm{B}_{-}$ & $\left\{\begin{array}{l}2 \mathrm{C}-\cdots \\
2 \mathrm{~F}-\cdots \\
2 \mathrm{~A} \cdots \\
2 \mathrm{~B} \cdots \\
2 \mathrm{D} \cdots \\
2 \mathrm{E} \cdots\end{array}\right.$ & $\begin{array}{r}70 \\
800 \\
800 \\
900 \\
900 \\
1,000 \\
1,000\end{array}$ & $\begin{array}{l}96,000 \text { E. L. } \\
42,000 \\
39,000 \\
32,000 \\
30,000 \\
9,000 \\
9,000\end{array}$ & $\begin{array}{r}135,500 \\
110,500 \\
113,000 \\
101,000 \\
101,000 \\
86,250 \\
87,000\end{array}$ & $\begin{array}{l}17.6 \\
16.5 \\
16.0 \\
17.0 \\
16.5 \\
17.5 \\
18.0\end{array}$ & $\begin{array}{l}54.9 \\
57.5 \\
55.6 \\
61.5 \\
63.5 \\
70.7 \\
72.0\end{array}$ & 281 \\
\hline E2457.. & $\left\{\begin{array}{l}\mathrm{B} 2- \\
\mathrm{B} 3- \\
\mathrm{B} 7- \\
\mathrm{B} 8 . \\
\mathrm{B} 6 . \\
\mathrm{B} 5- \\
39 .\end{array}\right.$ & $\begin{array}{r}70 \\
70 \\
700 \\
950 \\
950 \\
1,015 \\
1,075 \\
1,200 \\
1,200\end{array}$ & $\begin{array}{c}108,000 \text { E. L. } \\
109,500 \text { E. L. } \\
42,500 \\
30,000 \\
22,000 \\
13,000 \\
4,700 \\
2,250 \\
2,000\end{array}$ & $\begin{array}{r}135,500 \\
140,000 \\
122,500 \\
106,500 \\
107,000 \\
79,250 \\
64,000 \\
41,250 \\
38,250\end{array}$ & $\begin{array}{l}17.0 \\
17.2 \\
16.0 \\
19.0 \\
20.0 \\
25.0 \\
29.0 \\
32.5 \\
35.0\end{array}$ & $\begin{array}{l}40.7 \\
38.5 \\
35.0 \\
54.1 \\
52.5 \\
66.8 \\
74.1 \\
84.5 \\
85.9\end{array}$ & 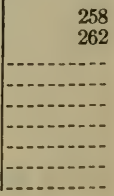 \\
\hline
\end{tabular}

s Elastic limit determined by The Midvale Co.

4 Tensile specimen was piped which resulted in a low tensile strength value. 
TABLE 2.-Tensile properties of materials tested in Table 1 at different temperatures-Continued

\begin{tabular}{|c|c|c|c|c|c|c|c|}
\hline Designation & Specimen No. & $\begin{array}{c}\text { Test } \\
\text { tempera- } \\
\text { ture }\end{array}$ & $\begin{array}{l}\text { Proportional } \\
\text { limit }\end{array}$ & $\begin{array}{l}\text { Tensile } \\
\text { strength }\end{array}$ & $\begin{array}{c}\text { Elonga- } \\
\text { tion in } 2 \\
\text { inches }\end{array}$ & $\begin{array}{l}\text { Reduc- } \\
\text { tion of } \\
\text { area }\end{array}$ & $\begin{array}{c}\text { Brinell } \\
\text { hardness } \\
\text { No. }\end{array}$ \\
\hline $7990 \ldots$ & & $\begin{array}{r}{ }^{\circ} F . \\
70 \\
1,500 \\
1,500\end{array}$ & $\begin{array}{l}\text { Lbs./in. }{ }^{2} \\
45,000 \mathrm{E.} \mathrm{L.}{ }^{3} \\
3,600 \\
3,900\end{array}$ & $\begin{array}{r}\text { Pbs. } / \text { in }^{2}{ }^{2} \\
108,000 \\
30,000\end{array}$ & $\begin{array}{r}\text { Per cent } \\
24.2 \\
29.0\end{array}$ & $\begin{array}{r}\text { Per cent } \\
43.7 \\
53.0\end{array}$ & \\
\hline FS-2, lot A. & & $\begin{array}{r}70 \\
990 \\
990 \\
1,200 \\
1,200 \\
1,360 \\
1,360\end{array}$ & $\begin{array}{r}68,000 \text { E. L. } \\
28,000 \\
28,000 \\
9,000 \\
11,000 \\
5,000 \\
6,000\end{array}$ & $\begin{array}{r}100,000 \\
83,500 \\
85,500 \\
60,000 \\
64,000 \\
38,000 \\
41,500\end{array}$ & $\begin{array}{l}33.0 \\
21.5 \\
24.5 \\
18.5 \\
19.0 \\
26.0 \\
22.5\end{array}$ & $\begin{array}{l}5.1 \\
31.4 \\
35.3 \\
37.5 \\
34.0 \\
50.3 \\
49.7\end{array}$ & 181 \\
\hline FS-2, lot B... & $\left\{\begin{array}{l}--- \\
3- \\
10- \\
5-- \\
9-- \\
2-- \\
7--\end{array}\right.$ & $\begin{array}{r}70 \\
990 \\
990 \\
1,200 \\
1,200 \\
1,360 \\
1,360\end{array}$ & $\begin{array}{l}54,000 \text { E. L. }{ }^{3} \\
28,000 \\
26,000 \\
8,000 \\
9,000 \\
4,500 \\
6,000\end{array}$ & $\begin{array}{l}99,500 \\
83,500 \\
87,000 \\
59,500 \\
62,300 \\
37,500 \\
37,500\end{array}$ & $\begin{array}{l}33.0 \\
21.5 \\
22.0 \\
22.5 \\
20.5 \\
24.5 \\
27.5\end{array}$ & $\begin{array}{l}47.7 \\
30.7 \\
34.7 \\
37.5 \\
37.5 \\
49.7 \\
54.4\end{array}$ & \begin{tabular}{l}
172 \\
\hdashline.- \\
\hdashline.- \\
$-\cdots$
\end{tabular} \\
\hline FS-1, lot A. & $\left\{\begin{array}{l}8-- \\
10 \\
9-- \\
2-. \\
4-. \\
3-. \\
1-.\end{array}\right.$ & $\begin{array}{r}70 \\
990 \\
890 \\
1,200 \\
1,200 \\
1,200 \\
1,360 \\
1,360\end{array}$ & $\begin{array}{r}63,000 \text { E. L. } \\
29,000 \\
30,000 \\
9,000 \\
14,000 \\
12,000 \\
6,500 \\
5,500\end{array}$ & $\begin{array}{r}112,500 \\
98,000 \\
97,000 \\
78,500 \\
72,500 \\
70,000 \\
46,500 \\
47,500\end{array}$ & $\begin{array}{l}30.0 \\
23.5 \\
24.0 \\
15.0 \\
13.0 \\
11.0 \\
10.0 \\
13.5\end{array}$ & $\begin{array}{l}36.9 \\
28.1 \\
27.1 \\
21.9 \\
21.2 \\
18.4 \\
16.9 \\
24.7\end{array}$ & 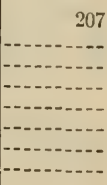 \\
\hline FS -1, lot B & $\left\{\begin{array}{l}2 \\
3 \\
3 \\
8 \\
8 \\
9 \\
5 \\
6 \\
11 \\
4\end{array}\right.$ & $\begin{array}{r}70 \\
990 \\
990 \\
1,200 \\
1,200 \\
1,200 \\
1,360 \\
1,360 \\
1,360 \\
1,360\end{array}$ & \begin{tabular}{c}
57,500 E. L. \\
27,000 \\
27,000 \\
9,800 \\
12,000 \\
\hdashline 1,000 \\
800 \\
\hdashline-
\end{tabular} & $\begin{array}{r}110,000 \\
95,000 \\
97,000 \\
79,000 \\
76,000 \\
66,000 \\
48,000 \\
50,500 \\
46,500 \\
45,000\end{array}$ & $\begin{array}{l}31.0 \\
25.0 \\
28.0 \\
16.5 \\
15.5 \\
14.0 \\
13.0 \\
12.5 \\
12.5 \\
12.5\end{array}$ & $\begin{array}{l}51.6 \\
22.6 \\
29.8 \\
21.2 \\
21.9 \\
19.1 \\
23.0 \\
20.5 \\
20.0 \\
21.2\end{array}$ & \begin{tabular}{r}
200 \\
\\
\hdashline \\
\\
\hdashline \\
\\
\\
\end{tabular} \\
\hline
\end{tabular}

Elastic limit determined by The Midvale Co

\section{MICROSTRUCTURE}

Any marked decrease in mechanical strength of alloys used continuously at elevated temperatures is usually reflected in the changed microstructure of the material. "Structural stability" at high temperature is of paramount importance in determining the usefulness of materials for such service. The microstructure of each of the alloys before and after testing was determined. In addition, the hardness was determined as a further indication of tempering which might have occurred during the heating The results of the hardness tests are summarized in Table 3 and the structures of the different alloys (except the plain carbon steel) are given in Figures 13 to 18, inclusive. The samples representative of the structure of the short-time test bars before heating were taken from the extreme butt ends of the tension bars (figs. 13, 14, 15, and 16) after the completion of the short-time test. The temperature at the ends of these bars was approximately $500^{\circ} \mathrm{F}$. lower than at the gage length of the bars and was assumed to be without effect on the original structure. In the case of Figures 16 and 17, the samples were taken from the butt ends of tension bars which had not been heated above room temperature. 
The samples representative of the alloys after heating (figs. 15 to 17) were taken at the center of the gage length of the bar close to the fracture in the case of the short-time tests and from a corresponding position (center of gage length) from the bars used in the flow tests. These latter bars were in the unfractured condition.

TABLE 3.-Hardness of the alloys used, before and after testing

\begin{tabular}{|c|c|c|c|c|c|c|c|c|}
\hline \multirow{3}{*}{ Designation of steel } & \multirow{2}{*}{\multicolumn{2}{|c|}{$\begin{array}{l}\text { Temperature } \\
\text { of test }\end{array}$}} & \multirow{3}{*}{$\begin{array}{c}\text { Type } \\
\text { of } \\
\text { test }\end{array}$} & \multicolumn{4}{|c|}{ Rockwell hardness } & \multirow{3}{*}{$\begin{array}{l}\text { Compare } \\
\text { figure and } \\
\text { micrograph }\end{array}$} \\
\hline & & & & \multicolumn{2}{|c|}{ Before test } & \multicolumn{2}{|c|}{ After test } & \\
\hline & ${ }^{\circ} \mathrm{F}$. & ${ }^{\circ} \mathrm{C}$. & & B & $\mathrm{C}$ & B & $\mathrm{C}$ & \\
\hline $\begin{array}{l}\text { 10-518, lot A- } \\
10-518 \text {, lot B.. } \\
\text { EE1455 } \\
\text { EE1456 } \\
\text { EE1454 }\end{array}$ & $\begin{array}{l}1,000 \\
1,000 \\
1,200 \\
1,200 \\
1,200\end{array}$ & $\begin{array}{l}540 \\
540 \\
650 \\
650 \\
650\end{array}$ & $\begin{array}{l}\text { S } 1 \\
\text { S } \\
\text { S } \\
\text { S } \\
\text { S }\end{array}$ & $\begin{array}{r}87 \\
95 \\
101\end{array}$ & $\begin{array}{r}-78 \\
25\end{array}$ & $\begin{array}{r}80 \\
90 \\
99\end{array}$ & $\begin{array}{l}26 \\
25\end{array}$ & $\begin{array}{l}\text { Fig. 14, } a, b \text {. } \\
\text { Fig. } 14, c, d \text {. } \\
\text { Fig. 13, } c, d . \\
\text { Fig. } 13, e, f . \\
\text { Fig. } 13, a, b .\end{array}$ \\
\hline 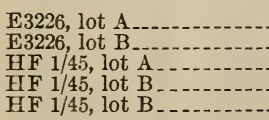 & $\begin{array}{l}1,000 \\
1,000 \\
1,000 \\
1,150 \\
1,000\end{array}$ & $\begin{array}{l}540 \\
540 \\
540 \\
620 \\
540\end{array}$ & $\begin{array}{l}S \\
S \\
S \\
S \\
S \\
S\end{array}$ & & $\begin{array}{l}36 \\
26 \\
46 \\
36 \\
42\end{array}$ & 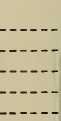 & $\begin{array}{l}35 \\
26 \\
46 \\
33 \\
31\end{array}$ & $\begin{array}{l}\text { Fig. } 14, e, f . \\
\text { Fig. } 14, g, h . \\
\text { Fig. } 15, a, b . \\
\text { Fig. } 15, c, d . \\
\text { Fig. } 15, e, f .\end{array}$ \\
\hline $\begin{array}{l}8 / 1317, \operatorname{lot} \mathrm{A} \\
8 / 1317, \operatorname{lot} \mathrm{B} \\
\mathrm{E} 2457\end{array}$ & $\begin{array}{l}1,000 \\
1,000 \\
1,200 \\
1,500\end{array}$ & $\begin{array}{l}540 \\
540 \\
650 \\
815\end{array}$ & $\begin{array}{l}S \\
S \\
S \\
S\end{array}$ & 98 & $\begin{array}{l}33 \\
29 \\
31\end{array}$ & 93 & $\begin{array}{l}33 \\
28 \\
27\end{array}$ & $\begin{array}{l}\text { Fig. } 16, a, b . \\
\text { Fig. } 16, c, d . \\
\text { Fig. } 16, e, f . \\
\text { Fig. } 16, g, h .\end{array}$ \\
\hline $\begin{array}{l}\text { FS-2, lot A. } \\
\text { FS-2, lot B. }\end{array}$ & $\left\{\begin{array}{l}1,360 \\
1,360 \\
1,360 \\
1,360\end{array}\right.$ & $\begin{array}{l}738 \\
738 \\
738 \\
738\end{array}$ & $\begin{array}{l}\mathrm{L}^{2} \\
\mathrm{~S} \\
\mathrm{~S}\end{array}$ & $\begin{array}{l}91 \\
95 \\
86 \\
93\end{array}$ & & $\begin{array}{l}83 \\
90 \\
79 \\
91\end{array}$ & & $\begin{array}{l}\text { Fig. } 17, a, b . \\
\text { Fig. } 17, c, d . \\
\text { Fig. } 17, e, f . \\
\text { Fig. } 17, g, h .\end{array}$ \\
\hline $\begin{array}{l}\text { FS-1, } \operatorname{lot} \mathrm{A}_{-} \\
\mathrm{FS}-1, \operatorname{lot} \mathrm{B}\end{array}$ & $\left\{\begin{array}{l}1,360 \\
1,360 \\
1,360 \\
1,360\end{array}\right.$ & $\begin{array}{l}738 \\
738 \\
738 \\
738\end{array}$ & $\begin{array}{l}\mathrm{L} \\
\mathrm{S} \\
\mathrm{L} \\
\mathrm{S}\end{array}$ & $\begin{array}{l}93 \\
97 \\
87 \\
91\end{array}$ & & $\begin{array}{l}85 \\
94 \\
85 \\
96\end{array}$ & -- & $\begin{array}{l}\text { Fig. } 18, a, b \text {. } \\
\text { Fig. } 18, c, d \text {. } \\
\text { Fig. } 18, e, f . \\
\text { Fig. } 18, g, h .\end{array}$ \\
\hline
\end{tabular}

\section{DISCUSSION}

The decided advantage of alloy steels over plain carbon steel for withstanding a load at elevated temperatures is very evident from the foregoing results. The proportional limit at elevated temperatures of the carbon steel used was very much lower than that of any of the alloy steels used at the corresponding temperature, and, furthermore, when stressed beyond the proportional limit, the rate at which the stress-strain curve for the alloy steels deviated from a straight line was much less than in the case of the carbon steels.

It is of interest to note in the two lots of chromium-vanadium steel (heat.10-518) which had been heat-treated differently so as to produce a decided difference in their tensile properties, as determined under ordinary conditions, that a marked difference in their tensile properties was maintained at temperatures up to $800^{\circ}$ to $1,000^{\circ} \mathrm{F}$. $\left(425^{\circ}\right.$ to $540^{\circ} \mathrm{C}$.). However, at the highest temperature used, $1,200^{\circ}$ F. $\left(650^{\circ}\right.$ C. $)$, the properties of the two lots were nearly the same nor was there any marked difference in the characteristics with respect to "flow."

The addition of tungsten to a chromium-vanadium steel in amounts of approximately 1.5 per cent raised the proportional limits of the steel at elevated temperatures. No marked improvement, however, 
HEAT NO. 10-918. C 0.40, MN 0.37, S1 0.23, CR 2.26, v 0.20 .

MOLLED BARS IST5F - I HA. AIR' $1300 \%$ - I IMR. TURNACE.

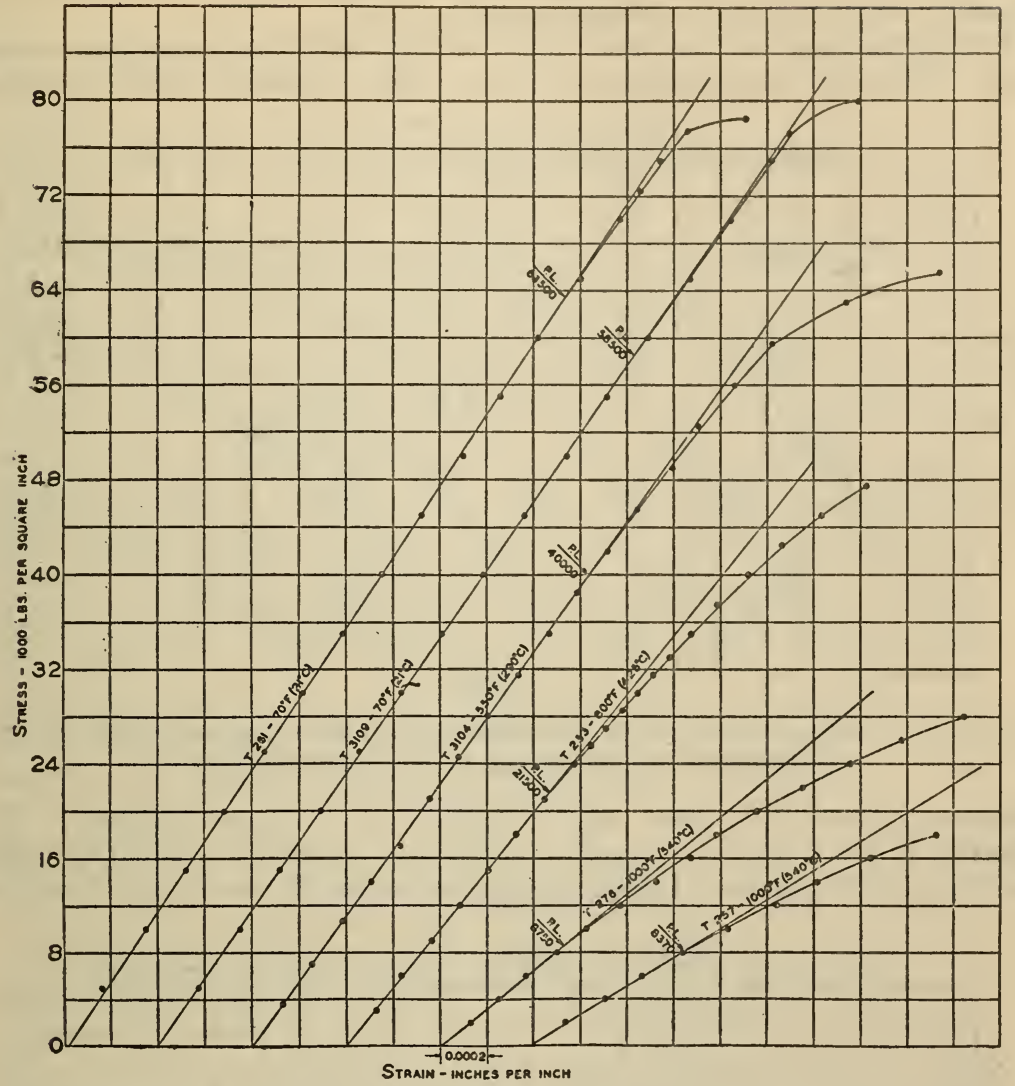

FIGURE 2.-Stress-strain curves of chrominum-vanadium steel tested in tension at different temperatures

HEAT MO EE 14Ss, COSS, UN 0.30, S1 0.16, CA 2.07, V 0.20, w 1.40.

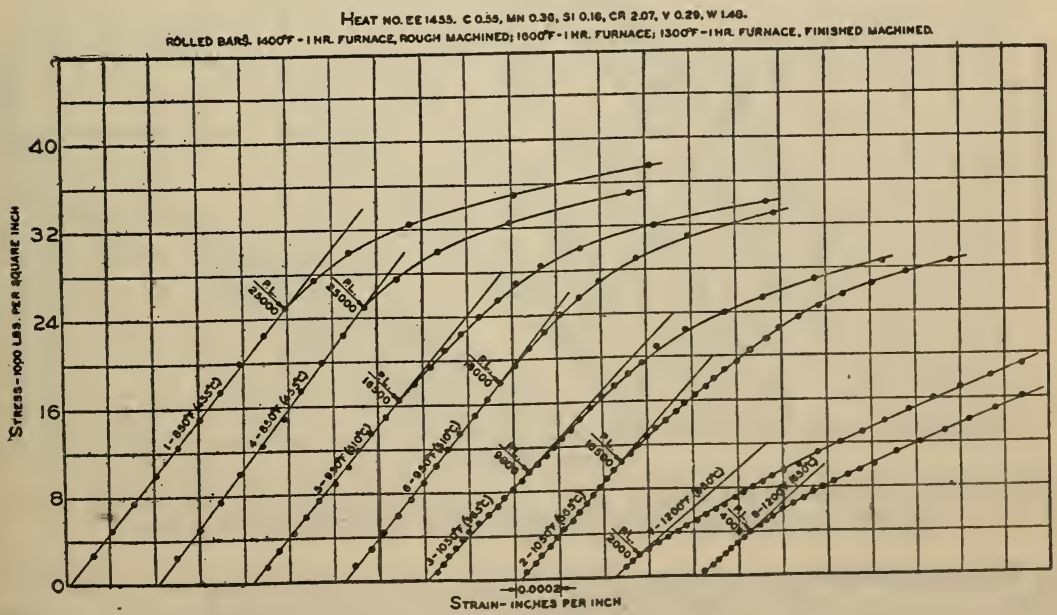

FigdRE 3.-Stress-strain curves of chromium-vanadium steel containing tungsten tested in tension at different temperatures 
resulted from the use, in addition to the tungsten, of another alloying element, such as silicon or aluminum.

A comparison of the two lots of the chromium-tungsten steel (heat $\mathrm{HF} 1 / 45$ ) containing 0.44 per cent carbon, 7.35 per cent

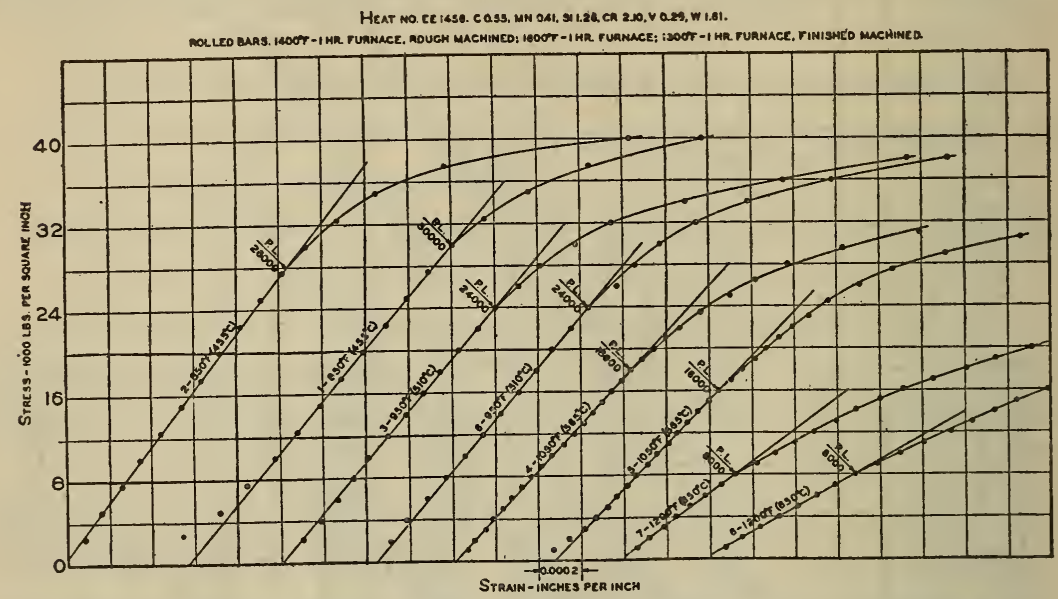

FIGURE 4.-Stress-strain curves of chromium-vanadium steel containing silicon and tungsten tested in tension at different temperatures

chromium, and 7.94 per cent tungsten is of some interest. The material of lot $\mathrm{A}$ had a proportional limit at $1,000^{\circ} \mathrm{F}$. $\left(540^{\circ} \mathrm{C}\right.$.) which was about 100 per cent higher than that of lot $\mathrm{B}$ due to the fact that

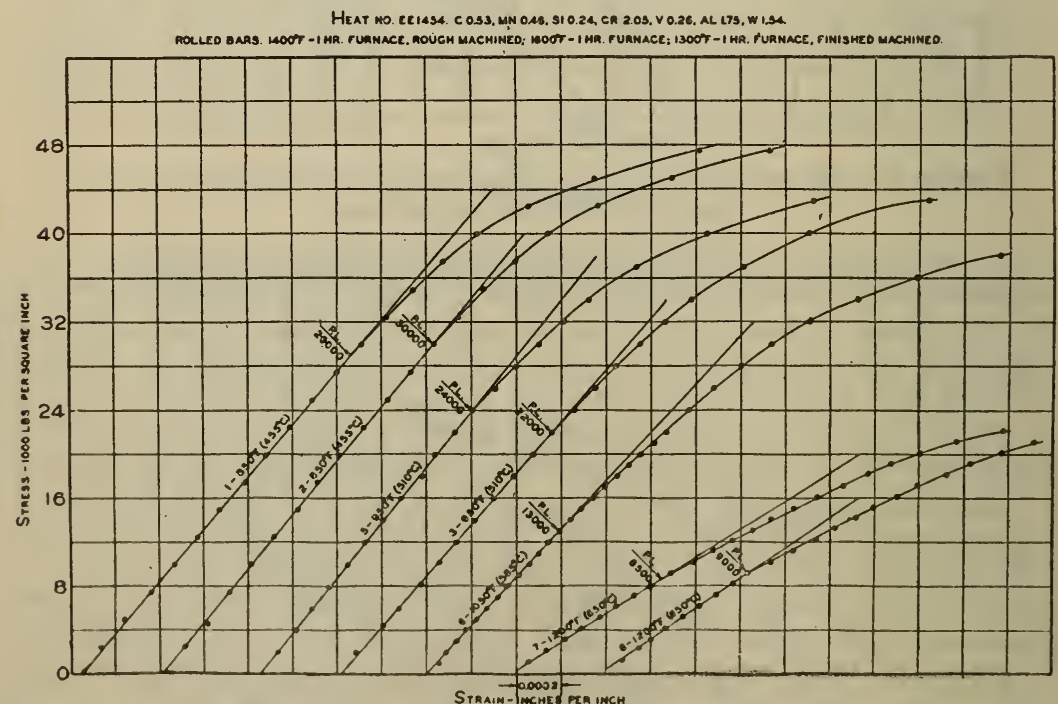

FIGURE 5.-Stress-strain curves of chromium-vanadium steel containing aluminum and tungsten tested in tension at different temperatures

the former had been water quenched before tempering at $1,200^{\circ} \mathrm{F}$. $\left(650^{\circ}\right.$ C. $)$, whereas lot $B$ was normalized before tempering at $1,200^{\circ}$ F. Likewise, lots $\Lambda$ and $B$ of the nickel-chormium-molybdenum 


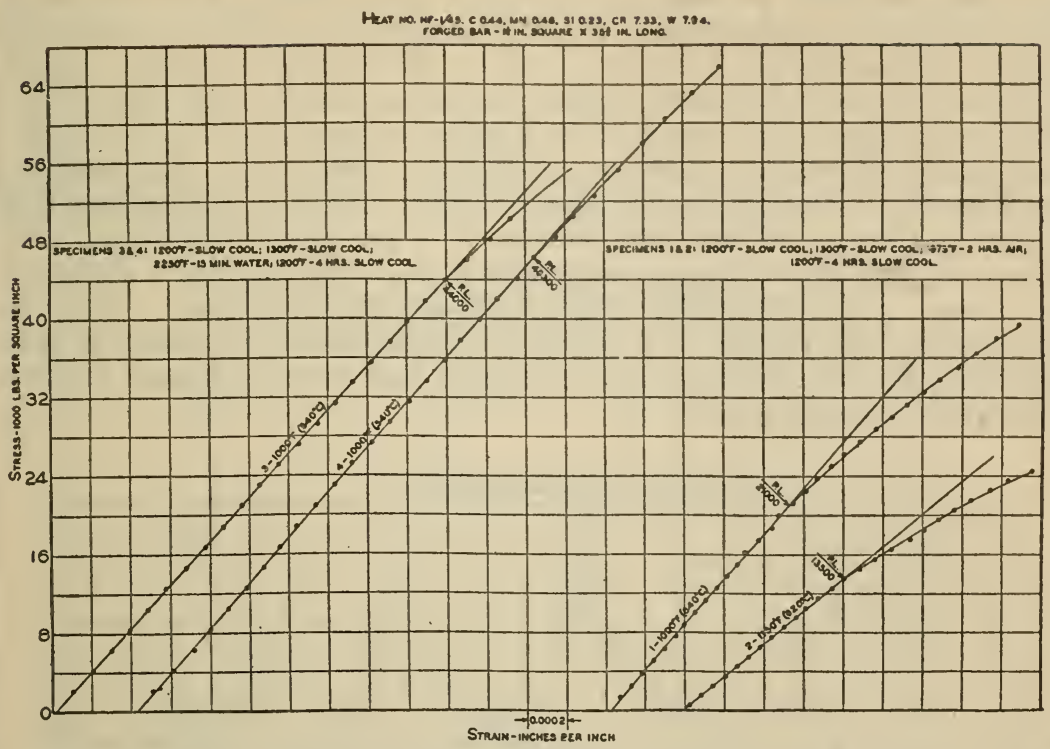

FIGURE 6.-Stress-strain curves of chromium-tungsten steel tested in tension at different temperatures

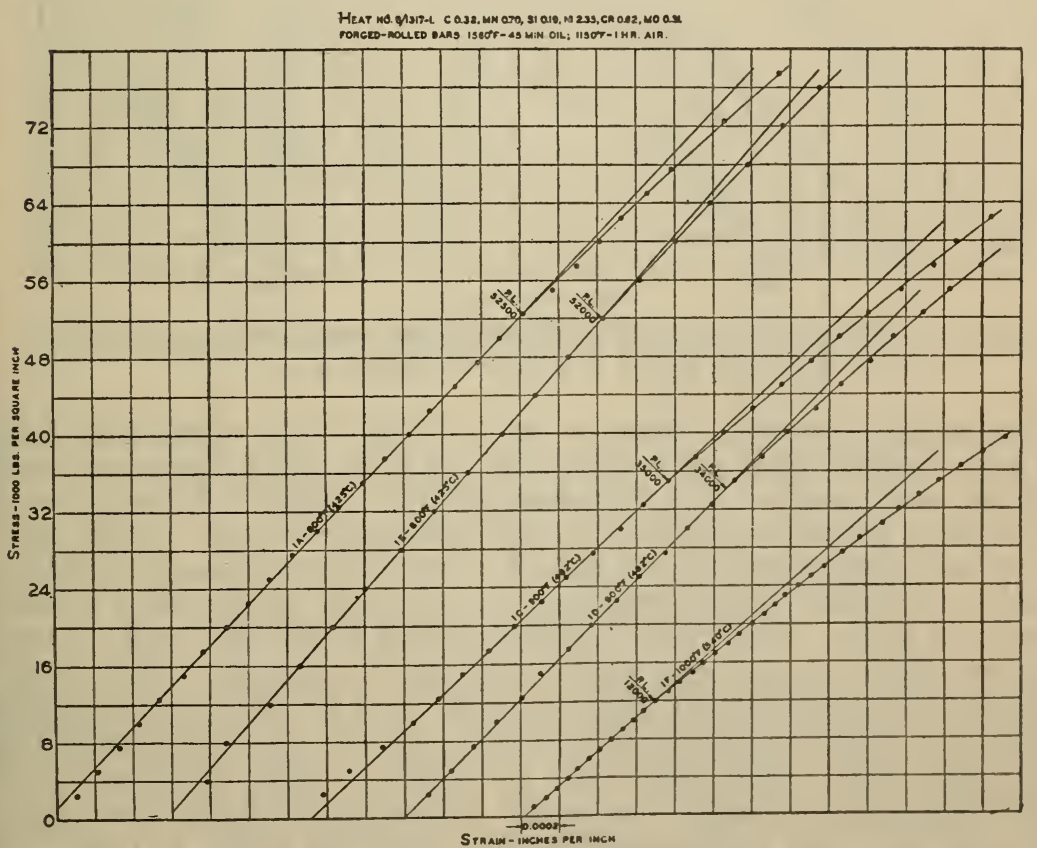

FígURE 7.-Stress-strain curves of nickel-chromium steel containing molybdenum tested in tension at different temperatures 
steel (heat 8/1317) containing 0.32 per cent carbon, 2.35 per cent nickel, 0.62 per cent chromium, and 0.31 per cent molybdenum showed different characteristics as determined by the short-time test. Lot A was quenched in oil before tempering while lot B was not. This difference in quenching resulted in higher proportional limits for lot A, especially at temperatures up to $900^{\circ} \mathrm{F}$. $\left(480^{\circ} \mathrm{C}\right.$.). The properties of the high chromium-nickel steel (heat E-2457) containing 0.39 per cent carbon, 1.50 per cent silicon, 10.5 per cent chromium, 1.61 per cent nickel, and 1.50 per cent aluminum were not very different from those of the nickel-chromium steel just mentioned. This is supported by the results of a previous publication ${ }^{18}$ in which it was found that $\mathrm{Fe}-\mathrm{Cr}-\mathrm{Ni}$ alloys low in nickel had low resistance to flow at elevated temperatures.

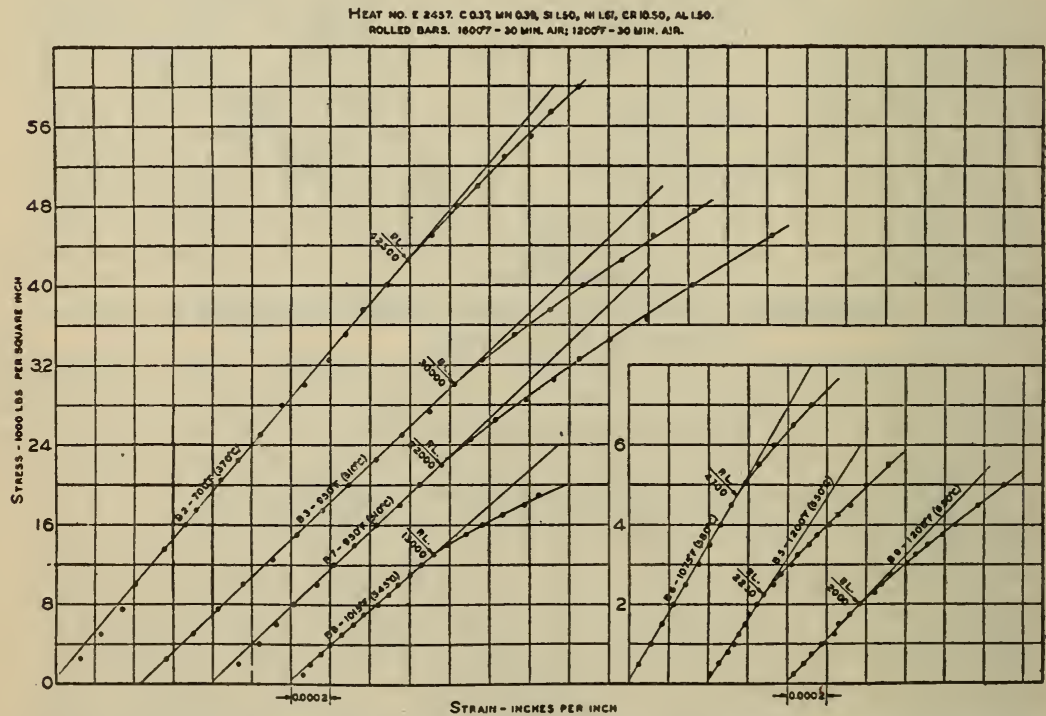

FIGURE 8.-Stress-strain curves of nickel-chromium steel containing aluminum Ttested in tension at different temperatures

The nickel-chromium-tungsten alloy (heat 7990) containing 0.53 per cent carbon, 26.0 per cent nickel, 14.8 per cent chromium, and 2.93 per cent tungsten was tested at $1,500^{\circ} \mathrm{F}$. $\left(820^{\circ}\right.$ C. $)$, and showed the highest proportional limit of all the alloys tested. This alloy was quenched in water from $1,835^{\circ} \mathrm{F}$. $\left(1,000^{\circ} \mathrm{C}\right.$.) and was, therefore, largely austenitic. In this respect it was similar to the other nickelchromium alloys (heat FS-2) containing 0.28 to 0.32 per cent carbon, 11.12 to 12.93 per cent chromium, and 34.9 to 35.9 per cent nickel, and the nickel-chromium-tungsten alloys (heat FS-1) containing 0.19 to 0.20 per cent carbon, 10.28 to 10.34 per cent chromium, 57.40 to 57.60 per cent nickel, and 3.19 to 3.30 per cent tungsten. The latter alloys gave nearly the same properties in both the shorttime and flow tests at temperatures of $1,200^{\circ}$ and $1,360^{\circ} \mathrm{F} .\left(650^{\circ}\right.$ and $738^{\circ}$ C.). These alloys have a strong tendency to retain austenite 
at both room and elevated temperatures, hence, their properties are not influenced greatly by previous heat treatments, such as annealing or quenching.

In expressing relative merits of steels intended for service at high temperatures, the stress required for causing a total "flow" or extension of 1 per cent or of 0.1 per cent at any given temperatures in 1,000 hours is commonly used. This has been done in Figure 12 which shows

HEAT NO. 7990. C 0.53, MN L58, SI 0.96, NI 26.00, CR 14.80, W 2.93.

FORGED-ROLLED BAR. $1835^{\circ} \mathrm{F}-1 \frac{1}{\mathrm{~S}}$ HR. WATER.

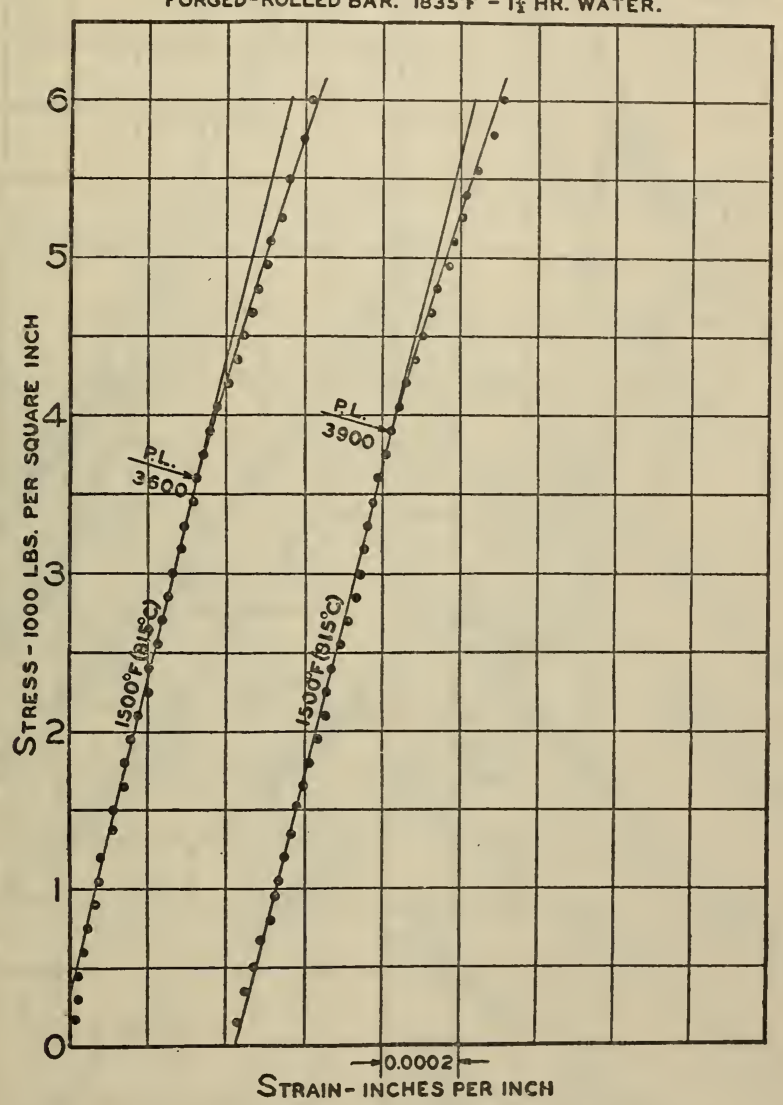

Figure 9.-Stress-strain curves of nickel-chromiumtungsten alloy with high nickel and chromium contents tested in tension at $1,500^{\circ} \mathrm{F} .\left(815^{\circ} \mathrm{C}\right.$. $)$

a comparison of the tensile properties, as determined by the short-time tension test and by the flow test, of two general classes of steel at elevated temperatures. The chromium-vanadium steel heat treated in two different ways, has been used as an example of one class, the pearlitic; the steels of high alloy content as representative of the austenitic class.

The question is often asked whether the short-time test can be used to replace the long, tedious, and expensive flow test. It will be noted 
(fig. 12) that in the case of the chromium-vanadium steels tested at $550^{\circ} \mathrm{F}$. $\left(285^{\circ}\right.$ C.), the lowest of the elevated temperatures used, the results indicated good agreement between the proportional limit determined at this temperature and the stress required to produce 0.1 per cent elongation at this same temperature in 1,000 hours. This relationship did not hold at higher temperatures up to $1,200^{\circ} \mathrm{F}$., however. The proportional limit determined by the short-time test at any given temperature was considerably higher than the

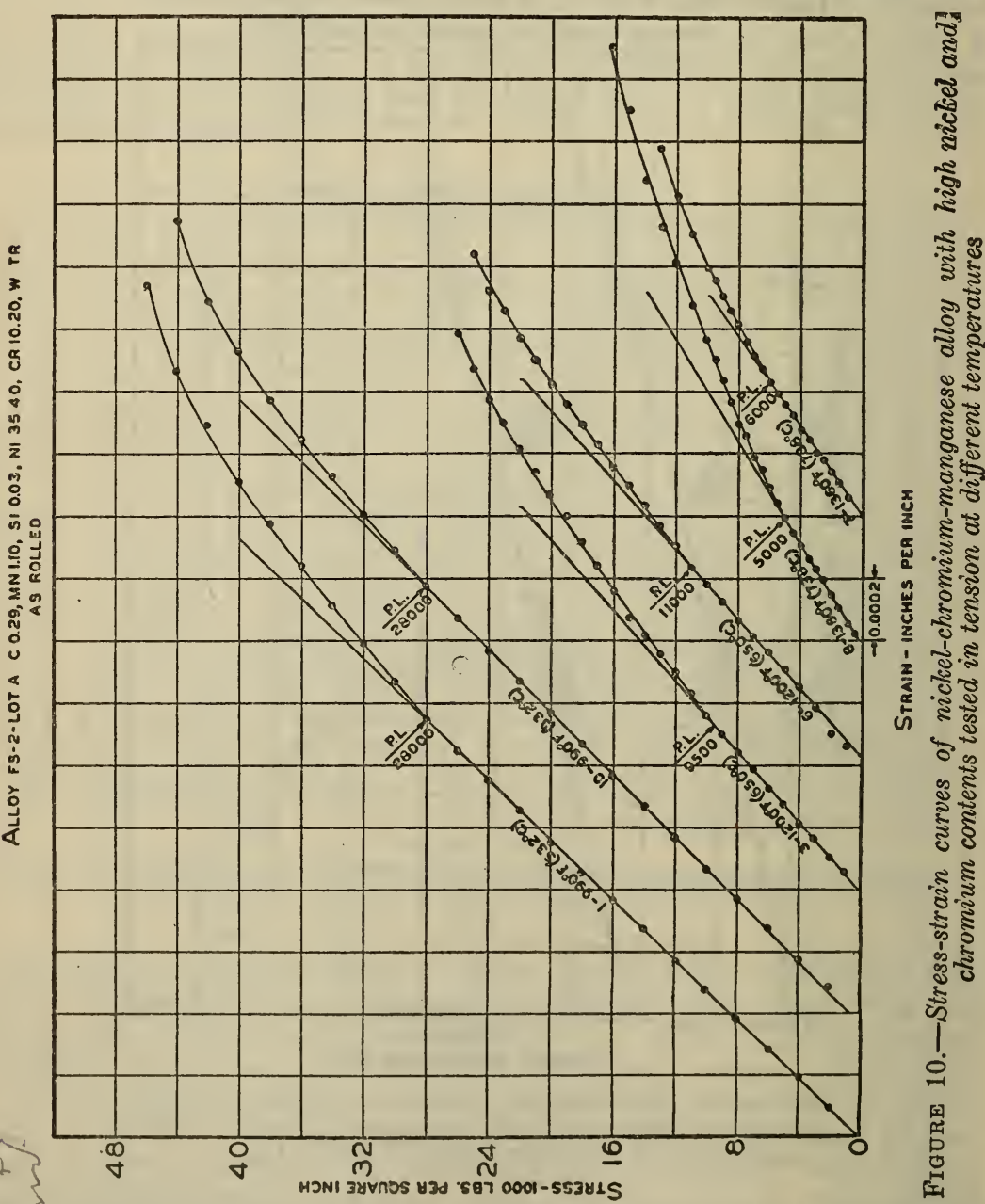

stress required to cause an extension of 1 per cent at that temperature in 1,000 hours. At the highest temperature used, $1,200^{\circ} \mathrm{F} .\left(650^{\circ} \mathrm{C}\right.$.), the stress required for 1 per cent extension in 1,000 hours was only very slightly higher than that required for an extension of 0.1 per cent and neither value differed appreciably from the proportional limit at this temperature.

In the case of the austenitic steels, the agreement between the results of the two tests was even less consistent. For one of the alloys used 


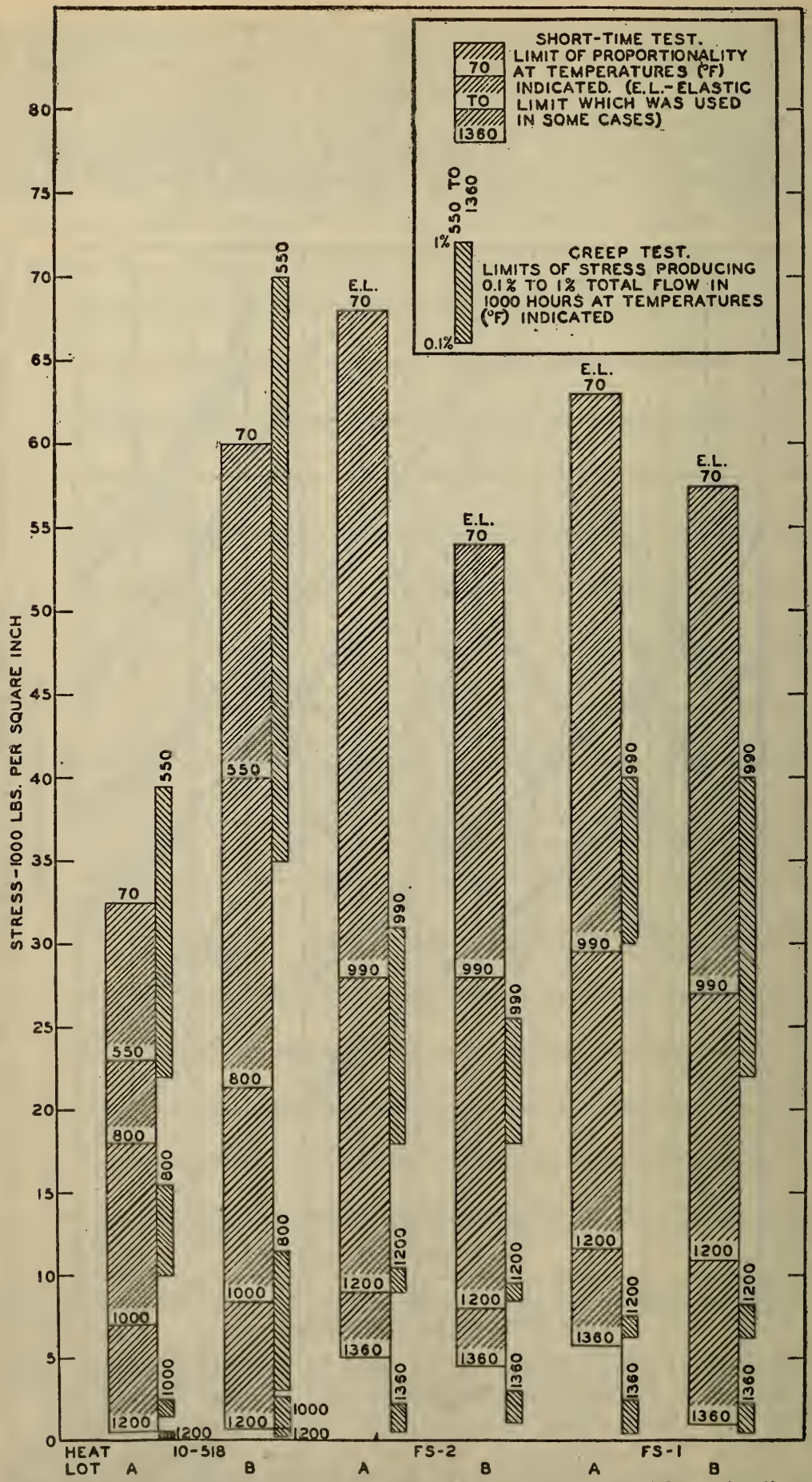

FIGURE 12.-Comparison of the results of "short-time" and "long-time" or creep tests of steels of the pearlitic and austenitic class

Seo Figures 2, 10, and 11. 


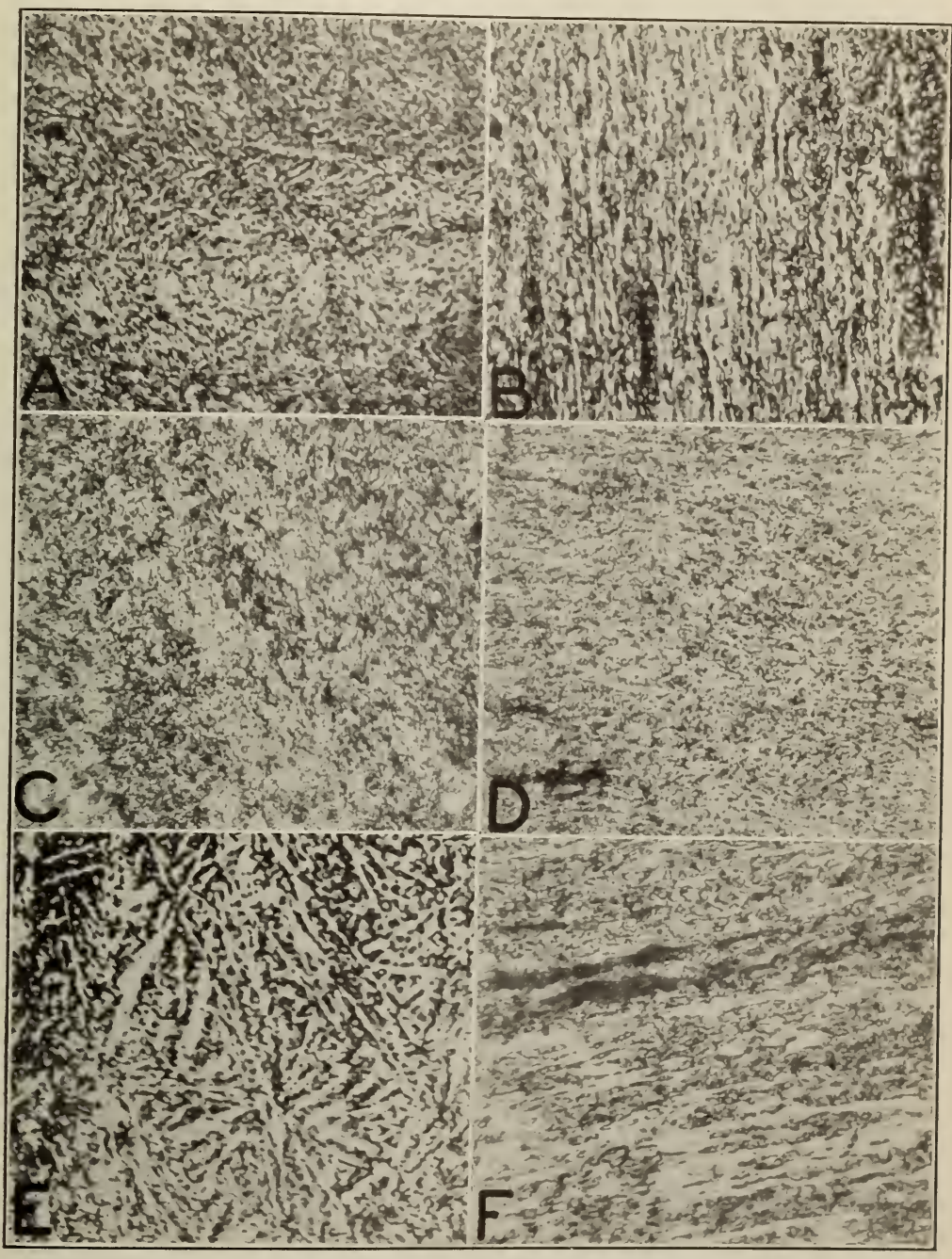

FIGURE 13.- Structure of chromium-vanadium-tungsten steel before and after testing in tension at $1,200^{\circ} \mathrm{F} .\left(650^{\circ} \mathrm{C}\right.$.) by the "short-time" method. $\times 500$

$A, C, E$, initial condition; $B, D, F$, after testing. (Sce Table 3.) $A, B$, chromium-vanadiumtungsten steel containing aluminum. (Ieat EE 1454, Table 1.) $C$, D, chromium-vanadiumtungsten steel containing silicon. (Heat E E 1455, Tablo 1.) E, F, chromium-vanadium-tungsten steel. (Heat EE 1456, Table 1.) The etching reagent used on the specimens shown here and in succeeding figures was aqua regia in glycerin. 


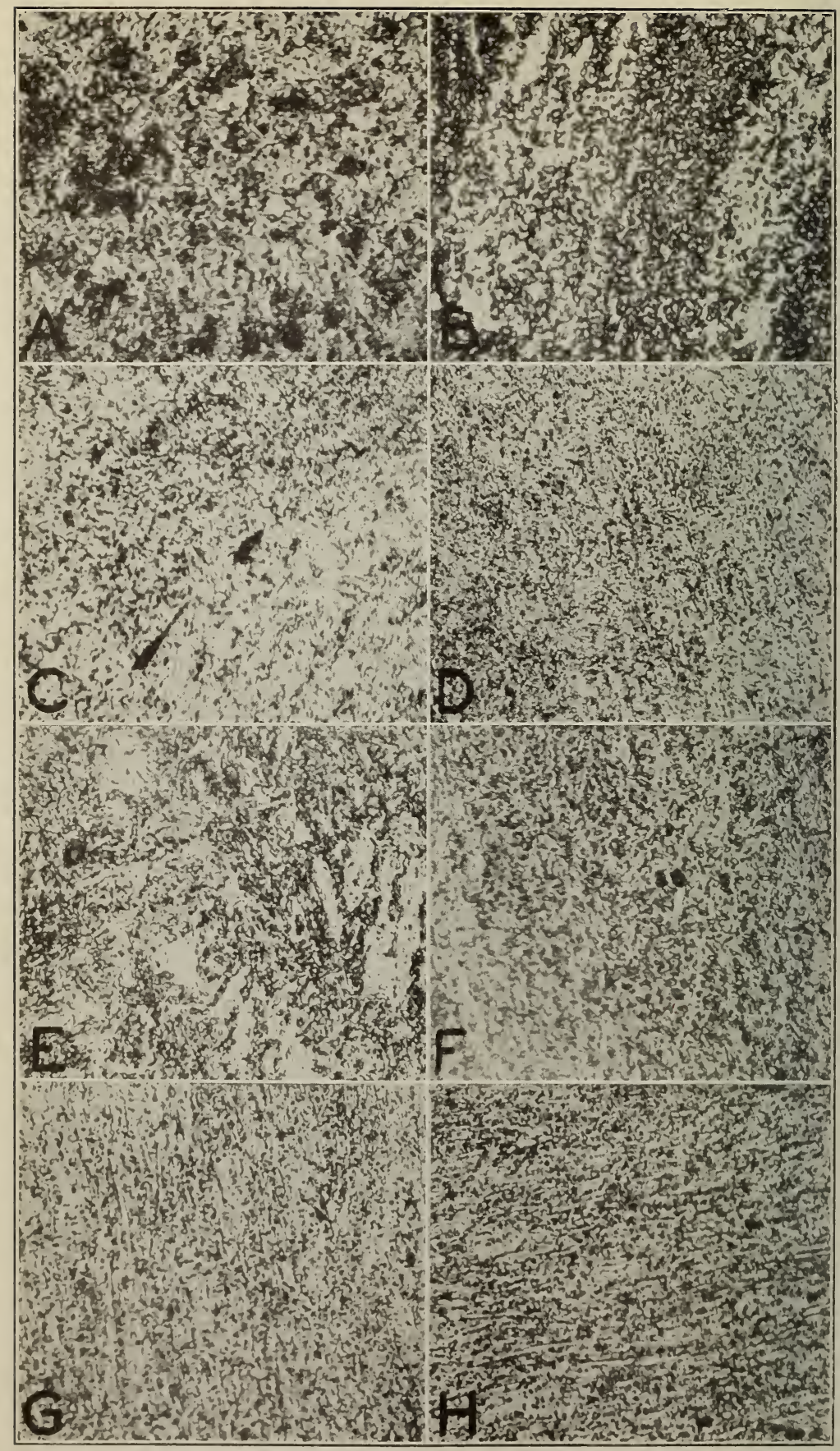

FIGURE 14.- Structure of chromium-ranadium steel and of chromium-vanadium-tungsten stecl before and after testing in tension at $1,000^{\circ} \mathrm{F} .\left(540^{\circ} \mathrm{C}\right.$.) by the "short-time" method. X 500

$A, C, E, G$, initial condition; $B, D, F, I I$, after testing. (See Table 3. ) $A, B, C, D$, chromiumvanadium stecl. (Ifeat 10-5is, lots $A$ and 13 , Table 1.) $E, F, G, I I$, chromium-vanadiumtungsten steel. (Heat E 3226 , lots $A$ and $\mathrm{B}$, Table 1.) 


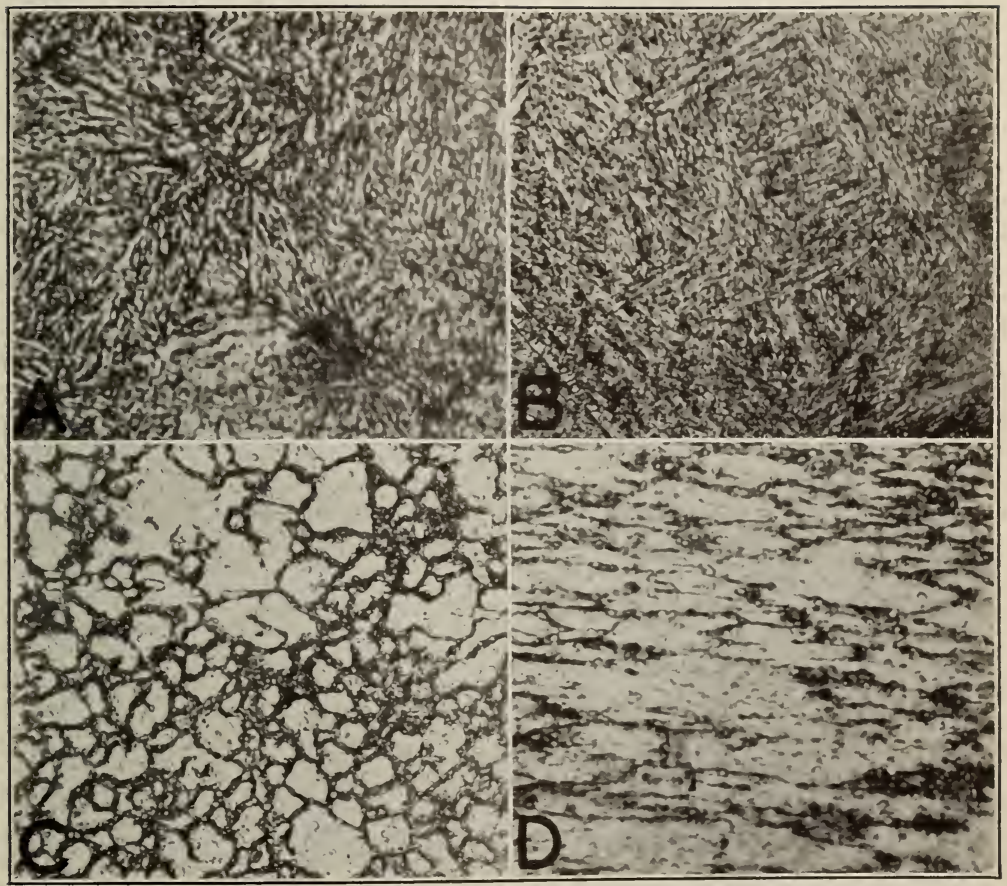

Frgure 15.-Structure of chromium-tungsten steel (heat IIF 1-4.5, lots $A$ and $B$, Table 1) before and after testing in tension at $1,000^{\circ} \mathrm{F} .\left(540^{\circ} \mathrm{C}.\right)$ by the "short-time" method. $\times 500$

$A, C$, initial condition; $B, D$, after testing. (Seo Table 3.) 


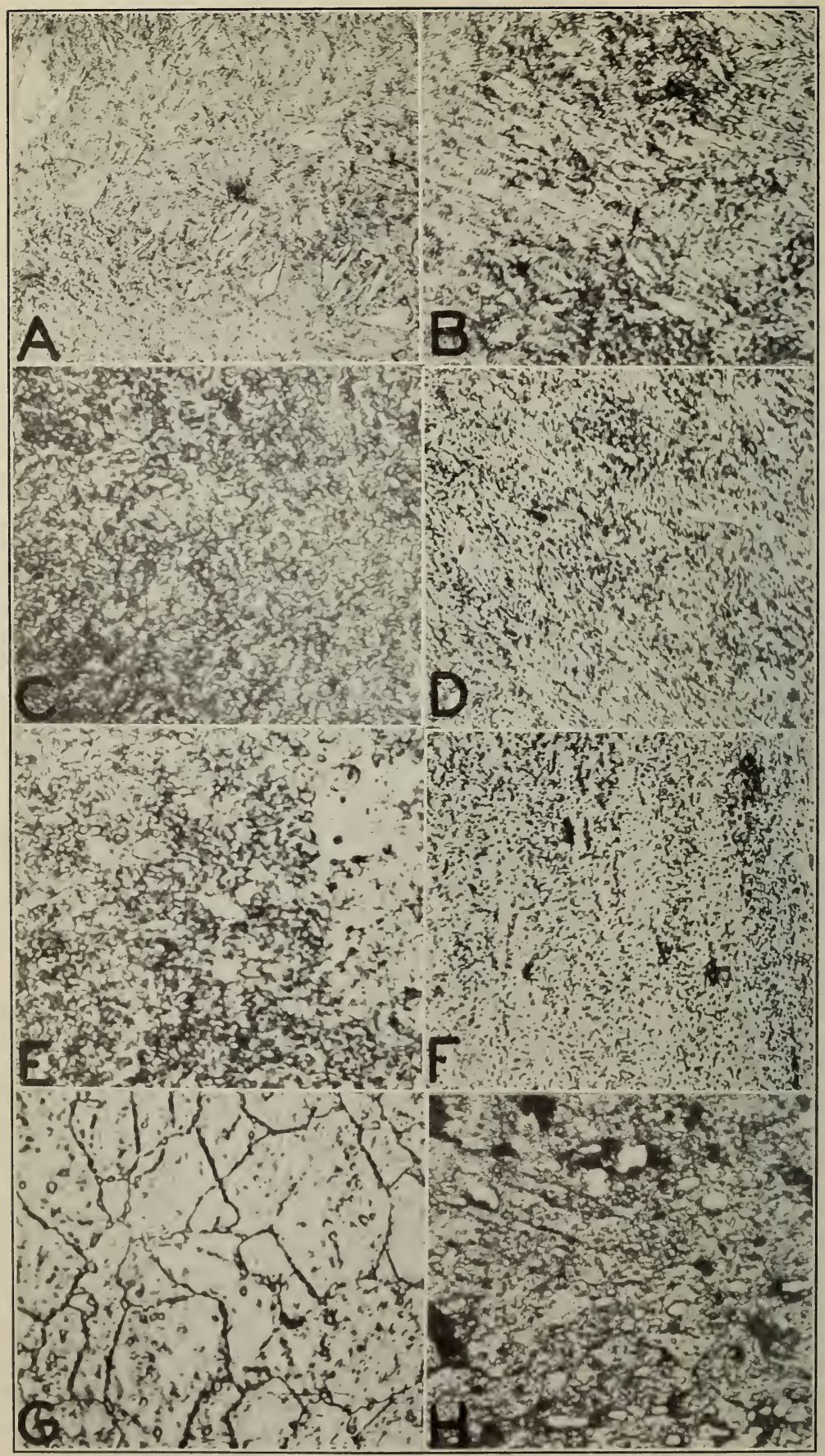

Figune 16. - Structure of various alloy stecls before and after testing in tension at clevated temperatures by the "short-time" test. $\times 500$

1. $C, E, G$, initial condition: $B, D, F, I I$, after testing. (See Tablo 3) $A, B, C, D$, nickelmolybdenum steel (heat $8-1317$, lots $\mathrm{A}$ and $\mathrm{B}$, Table 1) tested at $1,000^{\circ} \mathrm{F} .\left(510^{\circ} \mathrm{C}\right.$.). $E, F$, (chromium-nickel stecl containing aluminum (heat 2457 , Table 1) tested at $1,200^{\circ} \mathrm{F}$. $\left(650^{\circ} \mathrm{C}\right.$.). (i, II, chromium-nickel-tungsten stecl (heat 7990 , Table 1) tested at $1,500^{\circ} \mathrm{F} .\left(815^{\circ} \mathrm{C}\right.$.) 


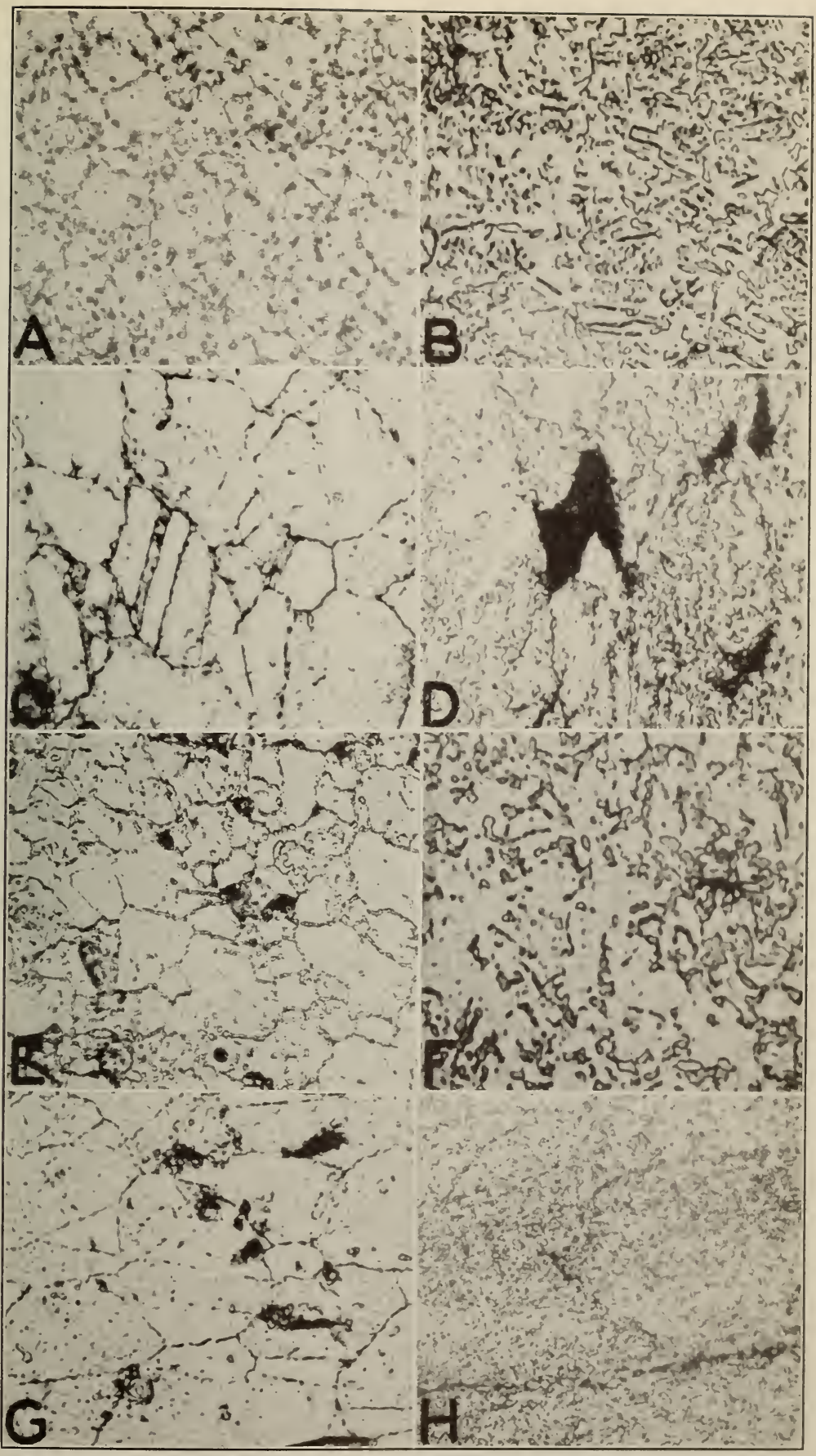

Figure 17.- Structure of chromium-nickel alloy (heat FS-2, lots $A$ and $B$,

Table 1) before and after testing in tension at $1,360^{\circ} \mathrm{F} .\left(735^{\circ} \mathrm{C}.\right)$. $\times 500$

$A, C, E, G$, initial condition; $B, D, F, I l$, after testing. (Sce Table 3.) $B$, after "long-time" or creep test; 335 hours, load 4,100 lbs./in. ${ }^{2}$, total extension 0.56 per cent. $D$, after short-time test. $F$, after creep test; 335 hours, load 4,100 lbs./in. ${ }^{2}$, total extension 0.80 per cent. $I I$, after short-time test. 


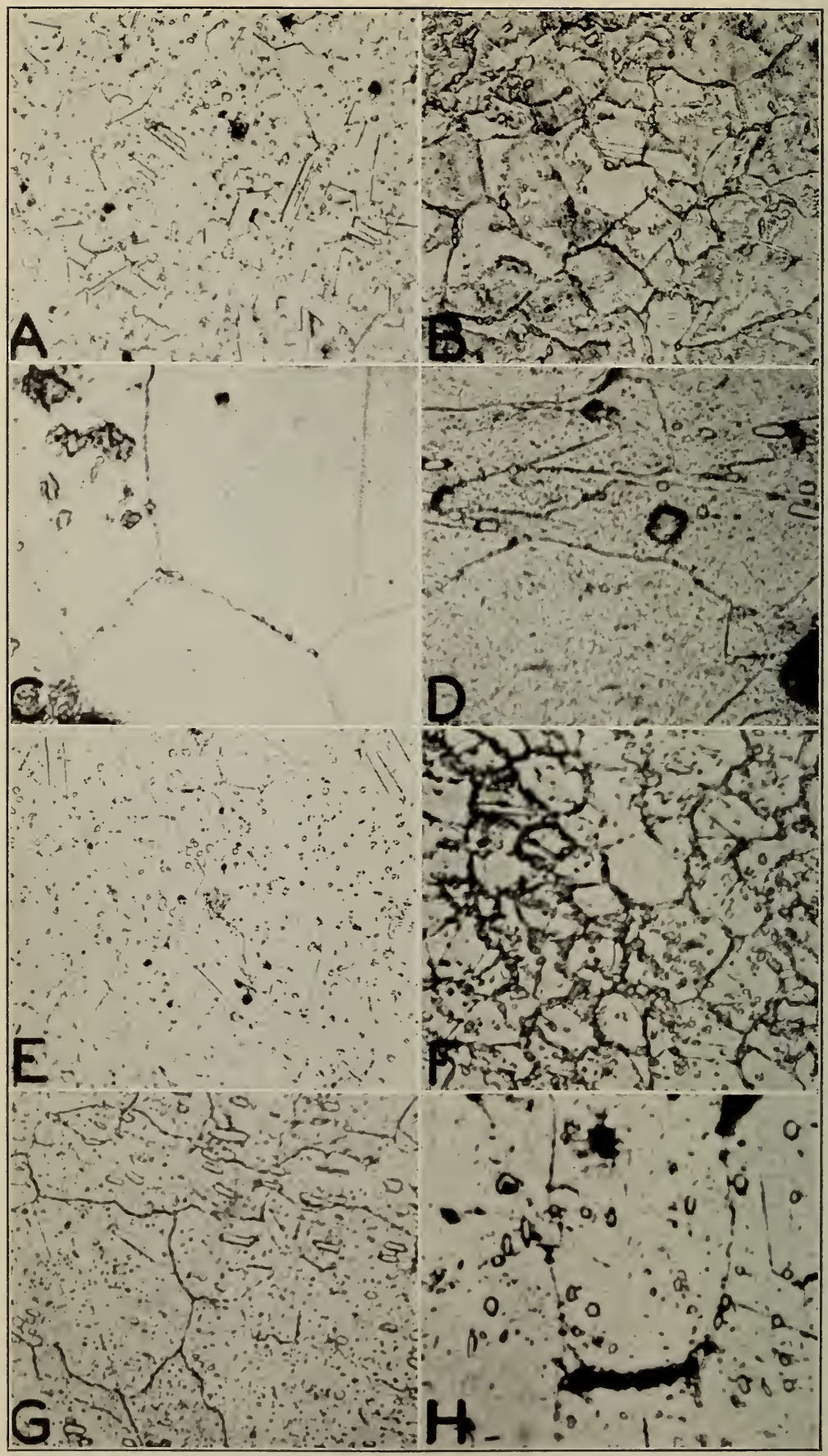

FIGURE 18.- Structure of chromium-nickel-tungsten alloy (heat FS-1, lots $A$ and $B$, Table 1 ), before and after testing in tension at $1,360^{\circ} \mathrm{F}$. $\left(735^{\circ} \mathrm{C}.\right) . \times 500$

$A, C, E, G$, initial condition; $B, D, F, I I$, after testing. (See Table 3.) B, after "long-time" or ereep test; 405 hours with load of $2,400 \mathrm{lbs}$./in. ${ }^{2}$, then 147 hours with 8,500 lbs./in. ${ }^{2}$, total elongation 2.6 per cent. $I$, after "short-time" test. $F$, after creep test, 405 hours with load of 2,500 lhs./in. ${ }^{2}$, then 147 hours with $8,500 \mathrm{lbs}$./in. ${ }^{2}$, total elongation, 3.4 per cent. II, after sohrt-time test. 
on account of the fact that the alloys were strained below their lower transformation temperature. This might be expected to increase the hardness somewhat, but it was found that in most of the materials represented by Figures 13, 14, and 15 there was a slight decrease in hardness after testing.

The medium alloy steels designated as 10-518, EE1455, EE1456, and 8/1317 appeared to be mostly sorbitic in structure (figs. 13, 14, and 15) while the high chromium-tungsten steel, HF-1/45, contained grains of sorbite with a network of troostite and some carbide constituent.

The high chromium steel E2457 (fig. 16), showed slight agglomeration of the cementitic constituent during the heating incidental to testing and the high nickel-chromium steel, 7990 (fig. 16), did likewise.

It is interesting to note that the nickel-chromium steel (FS-2) containing about 35 per cent nickel, 12 per cent chromium and 0.30 per cent carbon showed considerable agglomeration of the carbide constituent occurred (fig. 17) during both the "short-time" and the "long-time" tests. Before testing, these steels were essentially austenitic with small isolated particles of the carbide constituent. After testing these alloys at $1,360^{\circ} \mathrm{F}$. $\left(735^{\circ} \mathrm{C}\right.$.) , it was found that the lots in the "as rolled" state, and in the "rolled and annealed at $1,650^{\circ} \mathrm{F}$. $\left(900^{\circ} \mathrm{C}\right.$.)" state, showed considerable precipitation and agglomeration of the carbide constituent.

In the case of the higher nickel-chromium steel (FS-1) (fig. 18) containing about 57 per cent nickel, 10 per cent chromium, 3.25 per cent tungsten, and 0.20 per cent carbon, there appeared to be no marked change in structure with respect to the condition of the carbide constituent after similar treatments. It must be remembered that this alloy contains about 0.10 per cent less carbon than FS-2, but it is believed that the additional 22 per cent nickel, together with about 3.20 per cent tungsten is more likely to account for its increased stability as regards tempering.

\section{SUMMARY}

1. The tensile properties of a number of steels at a series of different elevated temperatures have been determined by means of the "short-time" test. The proportional limit was determined in each case. The steels used were a plain carbon steel, such as is used in boiler drums, and a number of alloy steels which have been considered in industry for service at high temperatures. The following were included: chromium-vanadium, chromium-vanadium-tungsten (the latter also with additions of silicon or aluminum) chromium-tungsten, nickel-moybdenum, and several austenitic steels containing nickel and chromium and, in some cases, tungsten.

2. The addition of tungsten, in amounts of 1.5 to 2 per cent, to a chromium-vanadium steel improves the tensile properties at high temperatures very decidedly. The further addition of silicon or aluminum does not appear to result in any marked improvement.

3. According to the short-time tests a marked increase in tensile properties of a steel brought about by heat treatment is maintained at elevated temperatures only so long as the temperature of testing 
is kept well below that used in tempering the steel in the preliminary heat treatment.

4. Both "short-time" tests and "flow" tests were carried out on several of the steels representative of the two classes-pearlitic and austenitic. According to the results, the "short-time" test can not satisfactorily replace the "flow" test in the evaluation of steels for service involving loads at high temperatures.

\section{ACKNOWLEDGMENTS}

The authors wish to express their appreciation to S. J. Rosenberg, associate metallurgist, and to S. E. Sinclair, junior metallurgist, of this bureau, for help in preparing many of the figures and micrographs.

Washington, October 15, 1930. 